Georgian Mathematical Journal

Volume 13 (2006), Number 1, 55-78

\title{
ON THE FOURIER EXPANSIONS OF EISENSTEIN SERIES OF SOME TYPES
}

\author{
NIKOLOZ KACHAKHIDZE
}

\begin{abstract}
Bases of the spaces of Eisenstein series $E_{k}\left(\Gamma_{0}(4 N), \chi\right)(k \in \mathbb{N}$, $k \geq 3, N$ is an odd natural and square-free $)$ and $E_{k / 2}\left(\widetilde{\Gamma}_{0}(4 N), \chi\right)(k \in \mathbb{N}$, $2 \nmid k, k \geq 5, N$ is an odd natural and square-free) are constructed for any Dirichlet character mod $4 N$ and Fourier expansions of these series are obtained.
\end{abstract}

2000 Mathematics Subject Classification: 11F11.

Key words and phrases: Entire modular form, Eisenstein series, congruence subgroup.

We will mostly use the notions and notation from [1]. Let $E_{k}\left(\Gamma_{0}(4 N), \chi\right)$ $(k \in \mathbb{N})$ denote the space of Eisenstein series of weight $k$ and character $\chi$ with respect to $\Gamma_{0}(4 N) ; E_{k / 2}\left(\widetilde{\Gamma}_{0}(4 N), \chi\right)(k \in \mathbb{N})$ denotes the space of Eisenstein series of weight $\frac{k}{2}$ and character $\chi$ with respect to $\widetilde{\Gamma}_{0}(4 N)$. Van Asch in [2] constructed a basis for the space $E_{k / 2}\left(\widetilde{\Gamma}_{0}(4 p),(\underline{4 p})\right)$ ( $p$ is an odd prime, $k \in \mathbb{N}$, $2 \nmid k ; k \geq 3$ if $p \geq 13 ; k \geq 5$ if $p=11 ; k \geq 7$ if $p=7 ; k \geq 9$ if $p=3$ or 5 ; $\left(\frac{4 p}{.}\right)$ is the Kronecker symbol) using theta-series of some positive quadratic forms. These series are given in the form of infinite products. Pei in [6] constructed bases for the spaces $E_{3 / 2}\left(\Gamma_{0}(4 N),\left(\frac{l}{?}\right)\right), E_{3 / 2}\left(\Gamma_{0}(8 N),\left(\frac{l}{2}\right)\right), E_{3 / 2}\left(\Gamma_{0}(8 N),\left(\frac{2 l}{)}\right)\right)$ and $E_{3 / 2}\left(\Gamma_{0}\left(2^{e}\right), 1\right), N$ being an odd natural and square-free number, $e$ an integer $\geq 4, l \mid N$, using transforms of some Eisenstein series of special kind and obtained Fourier expansions of these series.

In the present paper, for $N$ specified above, bases of the spaces $E_{k}\left(\Gamma_{0}(4 N), \chi\right)$ $(k \in \mathbb{N}, k \geq 3)$ and $E_{k / 2}\left(\widetilde{\Gamma}_{0}(4 N), \chi\right)(k \in \mathbb{N}, k \geq 5,2 \nmid k)$ are constructed for any character mod $4 N$ using the Eisenstein series, and their Fourier expansions are obtained.

In what follows, $p$ is an odd prime, $q$ is prime; $M, n, d, k, h, m, u, r, s, t$, $\alpha, \beta, \delta, \omega$ are integers; $\mathbb{H}=\{\tau \in \mathbb{C} \mid \operatorname{Im} \tau>0\}, z=\exp (2 \pi i \tau), \tau \in \mathbb{H} ;(m, n)$ denotes g.c.d. of $m$ and $n ; n$ mod $N$ means that $n$ runs through the full residue system modulo $N$; if $\tau \in \mathbb{C}$, then $\bar{\tau}$ denotes the complex conjugate of $\tau$. If $\chi$ is a character $\bmod N$, then $\chi_{0}$ denotes the main character $\bmod N$.

1. Lemma 1 ([4], p. 13). If $\chi(n)$ is a character $\bmod N$ and $N=N_{1}$. $N_{2} \cdots N_{m},\left(N_{r}, N_{s}\right)=1$ when $r \neq s$, then there is a unique system of characters $\chi_{1}, \chi_{2}, \ldots, \chi_{m}$ with modules $N_{1}, N_{2}, \ldots, N_{m}$, respectively, such that $\chi(n)=$ $\chi_{1}(n) \chi_{2}(n) \cdots \chi_{m}(n)$.

ISSN 1072-947X / \$8.00 / C Heldermann Verlag www.heldermann.de 
With mod 4 there are only two characters: the main character and the primitive character $\chi(n)=\left(\frac{-1}{n}\right)=(-1)^{(n-1) / 2}$ if $2 \nmid n$ and $\chi(n)=0$ if $2 \mid n$.

If $\chi$ is a character mod $p$, not the main character, then $\chi$ is primitive (see [4], p. 22). With $\bmod p$ there exists only one real primitive character $\chi(n)=\left(\frac{n}{p}\right)$ $\left(\left(\frac{n}{p}\right)\right.$ is the generalized Legendre symbol).

In what follows $\phi(n)$ denotes a character $\bmod 4$. If $\chi$ is a character $\bmod N$, then let

$$
g(\chi)=\frac{1}{\sqrt{N}} \sum_{n \bmod N} \chi(n) \exp (2 \pi i n / N) .
$$

Lemma 2 ([4], p. 45). If $\chi$ is a primitive character $\bmod N$, then

$$
\sum_{n \bmod N} \chi(n) \exp (2 \pi i m n / N)=\bar{\chi}(m) \sqrt{N} g(\chi), \quad|g(\chi)|=1
$$

Lemma 3 ([4], p. 50). If $\chi$ is a real primitive character mod $N$, then

$$
g(\chi)= \begin{cases}1 & \text { when } \chi(-1)=1 \\ i & \text { when } \chi(-1)=-1\end{cases}
$$

Lemma $4\left([4]\right.$, p. 52). Let $\chi(n)=\chi_{1}(n) \chi_{2}(n)$, where $\chi_{r}$ is a character $\bmod N_{r}, r=1,2$, and $\left(N_{1}, N_{2}\right)=1$, then

$$
\begin{aligned}
\sum_{n \bmod N} \chi(n) \exp (2 \pi i m n / N)=\chi_{1}\left(N_{2}\right) \chi_{2}\left(N_{1}\right) & \sum_{n_{1} \bmod N_{1}} \chi_{1}\left(n_{1}\right) \exp \left(2 \pi i m n_{1} / N_{1}\right) \\
& \times \sum_{n_{2} \bmod N_{2}} \chi_{2}\left(n_{2}\right) \exp \left(2 \pi i m n_{2} / N_{2}\right) .
\end{aligned}
$$

Using Lemma 2 it is easy to verify (see [1], Ch. IV, §2, [4], p. 39) that if $\psi$ is a character $\bmod p$, then

$$
\begin{aligned}
& \sum_{r \bmod p} \psi(r) \exp \left(2 \pi i n r / p^{\beta}\right)=0 \text { if } \psi \neq \psi_{0}, \quad p^{\beta} \mid n ; \\
& p-1 \text { if } \psi=\psi_{0}, p^{\beta} \mid n \text {; } \\
& -1 \text { if } \psi=\psi_{0}, \quad p^{\beta} \nmid n, p^{\beta-1} \mid n \text {; } \\
& \sqrt{p} \bar{\psi}\left(n / p^{\beta-1}\right) g(\psi) \text { if } \psi \neq \psi_{0}, p^{\beta} \nmid n, p^{\beta-1} \mid n \text {. } \\
& \begin{aligned}
\sum_{r \bmod 4} \phi(r) \exp \left(2 \pi i n r / 2^{\alpha}\right)= & 0 \text { if } \phi \neq \phi_{0}, \quad 2^{\alpha-1} \mid n ; \\
& 0 \text { if } \phi=\phi_{0}, \quad 2^{\alpha-2} \mid n, \quad 2^{\alpha-1} \nmid n ;
\end{aligned} \\
& 2 \text { if } \phi=\phi_{0}, 2^{\alpha} \mid n \text {; } \\
& -2 \text { if } 2^{\alpha-1} \mid n, \phi=\phi_{0}, 2^{\alpha} \nmid n \text {; } \\
& 2 i \phi\left(n / 2^{\alpha-2}\right) \text { if } \phi \neq \phi_{0}, 2^{\alpha-2} \mid n, 2^{\alpha-1} \nmid n \text {. } \\
& \sum_{r \bmod \omega} \exp (2 \pi i n r / \omega)= \begin{cases}\omega & \text { if } \omega \mid n ; \\
0 & \text { if } \omega \nmid n .\end{cases}
\end{aligned}
$$


Lemma 5 ([1], Ch. IV, §2). If $\tau \in \mathbb{H}, a \in \mathbb{R}, a>1$, then

$$
\sum_{h=-\infty}^{+\infty}(\tau+h)^{-a}=(2 \pi)^{a} \exp (-\pi i a / 2)(\Gamma(a))^{-1} \sum_{n=1}^{+\infty} n^{a-1} \exp (2 \pi i \tau n)
$$

where $\Gamma$ is the Euler function and when $\tau \in \mathbb{H}, \tau^{a}=\exp (a \ln \tau) ;$ also $\ln \tau$ denotes the branch of logarithm for which $0<\operatorname{Im}(\ln \tau)<\pi$.

Let $\sigma_{\infty}$ denote the number of cusps with respect to $\Gamma_{0}(N)$. Then (see [5], p. 102)

$$
\sigma_{\infty}=\sum_{t \mid N} \varphi((t, N / t))
$$

where $\varphi$ is the Euler function.

Lemma 6. If $N=p_{1} \cdots p_{j}$, then $\Gamma_{0}(4 N)$ has $3 \cdot 2^{j}$ cusps: $\zeta_{1}=\infty, \sigma_{1}=\left(\begin{array}{cc}1 & 0 \\ 0 & 1\end{array}\right)$; $\zeta_{2}=0, \sigma_{2}=\left(\begin{array}{cc}0 & 1 \\ -1 & 0\end{array}\right) ;\left\{\zeta=-\frac{1}{N_{1}}, \sigma=\left(\begin{array}{cc}1 & 0 \\ -N_{1} & 1\end{array}\right)\left|N_{1}\right| 4 N, N_{1} \neq 1, N_{1} \neq 4 N\right\}$; $\zeta_{r}=\sigma_{r} \infty(r=1,2), \zeta=\sigma \infty$.

Proof directly follows from (1.4) and the definition of a cusp.

Lemma 7 ([1], Ch. IV, §2). If $\omega=\omega_{0} \omega_{1}^{2}, 2 \nmid \omega, \omega_{0}$ and $n$ are square-free, then

$$
\sum_{r \bmod \omega}\left(\frac{r}{\omega}\right) \exp (2 \pi i n r / \omega)= \begin{cases}0 & \text { when } \omega_{1} \nmid n ; \\ \varepsilon_{\omega}\left(\frac{n}{\omega_{0}}\right) \sqrt{\omega_{0}} \mu\left(\omega_{1}\right) \omega_{1} & \text { when } \omega_{1} \mid n\end{cases}
$$

where

$$
\varepsilon_{\omega}= \begin{cases}1 & \text { if } \omega \equiv 1(\bmod 4) \\ i & \text { if } \omega \equiv-1(\bmod 4)\end{cases}
$$

$\left(\frac{r}{\omega}\right)$ is the generalized Jacobi symbol and $\mu$ is the Möbius function.

In what follows, let $\chi$ be the Dirichlet character $\bmod N ; \zeta=\sigma \infty, \sigma \in$ $S L_{2}(\mathbb{Z}), \Gamma_{\zeta}=\left\{\gamma \in \Gamma_{0}(N) \mid \gamma \zeta=\zeta\right\}$; if $\gamma=\left(\begin{array}{cc}a & b \\ c & d\end{array}\right) \in S L_{2}(\mathbb{Z}), \tau \in \mathbb{H}, k \in \mathbb{N}$, let $J_{k}(\gamma, \tau)=(c \tau+d)^{k},\left.f(\tau)\right|_{k} \gamma=J_{k}(\gamma, \tau)^{-1} f(\gamma \tau)$.

Lemma 8 (see [1], Ch. III, §2; [3], Ch. II, §1). a) Let $\chi(-1)=(-1)^{k}$,

$$
E(\tau ; k, N, \chi)=\sum_{\gamma \in \Gamma_{\zeta} \backslash \Gamma_{0}(N)} \bar{\chi}(d) J_{k}\left(\sigma^{-1} \gamma, \tau\right)^{-1},
$$

where $\Gamma_{\zeta} \backslash \Gamma_{0}(N)$ denotes the set of right cosets of $\Gamma_{0}(N)$ by $\Gamma_{\zeta}$. Then $E(\tau ; k, N, \chi) \in M_{k}\left(\Gamma_{0}(N), \chi\right)$ for any $k \geq 3$.

b) If $E(\tau ; k, N, \chi) \not \equiv 0$, then $E(\tau ; k, N, \chi) \neq 0$ only at the cusp $\zeta$ and vanishes at the remaining cusps.

In what follows, let $\tau^{k / 2}=(\sqrt{\tau})^{k},-\frac{\pi}{2}<\operatorname{Arg} \sqrt{\tau} \leq \frac{\pi}{2}$ for any $k \in \mathbb{Z}$; if $\gamma=\left(\begin{array}{ll}a & b \\ c & d\end{array}\right) \in S L_{2}(\mathbb{Z}), \tau \in \mathbb{H}$, suppose $\varphi(\gamma, \tau)$ to be a holomorphic function on $\mathbb{H}$ such that $\varphi^{2}(\gamma, \tau)=t(c \tau+d), t \in\{-1 ; 1\}$. If $\gamma \in \Gamma_{0}(4)$, then $\varphi(\gamma, \tau)=$ $j(\gamma, \tau)=\left(\frac{c}{d}\right) \varepsilon_{d}^{-1} \sqrt{c \tau+d}$, where $\varepsilon_{d}$ is defined by (1.5), $\left(\frac{c}{d}\right)$ is the generalized 
Jacobi symbol when $d$ is odd positive and if $d$ is odd negative, then $\left(\frac{c}{d}\right)=$ $\operatorname{sgn} c\left(\frac{c}{|d|}\right)$, also $\left(\frac{0}{ \pm 1}\right)=1$.

Let $G=\left\{(\gamma, \varphi(\gamma, \tau)) \mid \gamma \in S L_{2}(\mathbb{Z})\right\}$. If $\left(\gamma_{1}, \varphi\left(\gamma_{1}, \tau\right)\right),\left(\gamma_{2}, \varphi\left(\gamma_{2}, \tau\right)\right) \in G$, suppose $\left(\gamma_{1}, \varphi\left(\gamma_{1}, \tau\right)\right) \cdot\left(\gamma_{2}, \varphi\left(\gamma_{2}, \tau\right)\right)=\left(\gamma_{1} \gamma_{2}, \varphi\left(\gamma_{1}, \gamma_{2} \tau\right) \varphi\left(\gamma_{2}, \tau\right)\right)$. It is known (see [1], Ch. IV, §1) that $G$ is a group with respect to this operation.

If $\xi=(\gamma, \varphi(\gamma, \tau)) \in G, f$ is some function defined on $\mathbb{H}$, let $\left.f(\tau)\right|_{k / 2} \xi=$ $\varphi(\gamma, \tau)^{-k} f(\gamma \tau)$. If $4 \mid N$, suppose $\widetilde{\Gamma}_{0}(N)=\left\{(\gamma, j(\gamma, \tau)) \mid \gamma \in \Gamma_{0}(N)\right\}$.

Lemma 9 (see [1], Ch. IV, $\S 2 ;[3]$, Ch. II, $\S 1$ ). a) Let $4 \mid N, \chi(-1)=1$,

$$
E\left(\tau ; \frac{k}{2}, N, \chi\right)=\sum_{\gamma \in \Gamma_{\zeta} \backslash \Gamma_{0}(N)} \bar{\chi}(d) \varphi\left(\sigma^{-1} \gamma, \tau\right)^{-k}
$$

where $\gamma=\left(\begin{array}{ll}a & b \\ c & d\end{array}\right)$. Then $E\left(\tau ; \frac{k}{2}, N, \chi\right) \in M_{k / 2}\left(\widetilde{\Gamma}_{0}(N), \chi\right)$ for any $k \geq 5$.

b) $E\left(\tau ; \frac{k}{2}, N, \chi\right) \neq 0$ only at the cusp $\zeta$ and vanishes at the remaining cusps.

In the following let

$$
\begin{gathered}
\rho_{r}(u, \chi)=\sum_{\delta d=u} \chi(\delta) d^{r}, \quad \sigma_{r}(u)=\sum_{d \mid u} d^{r} ; \\
\mathcal{L}(k, \chi)=\sum_{n=1}^{\infty} \chi(n) n^{-k}=\prod_{q}\left(1-\chi(q) q^{-k}\right)^{-1} ; \\
\mathcal{L}\left(\frac{k-1}{2},(-1)^{\frac{k-1}{2}} u\right)=\sum_{n=1}^{\infty}\left(\frac{(-1)^{\frac{k-1}{2}} u}{n}\right) n^{\frac{1-k}{2}} \\
=\prod_{q>2}\left(1-\left(\frac{(-1)^{\frac{k-1}{2}} u}{q}\right) q^{\frac{1-k}{2}}\right)^{-1} \text { (Dirichlet } \mathcal{L} \text {-function); } \\
\zeta(k)=\sum_{n=1}^{\infty} n^{-k}=\prod_{q}\left(1-q^{-k}\right)^{-1} \text { (Riemann } \zeta \text {-function). }
\end{gathered}
$$

2. In this section let $N=p_{1} p_{2} \cdots p_{j}, n=2^{s} p_{1}^{t_{1}} p_{2}^{t_{2}} \cdots p_{j}^{t_{j}} u,(2 N, u)=1$. It follows from Lemma 1 that if $\chi(n)$ is a character $\bmod 4 N$, then $\chi(n)=$ $\phi(n) \psi(n)=\phi(n) \psi_{1}(n) \cdots \psi_{j}(n)$, where $\psi(n)$ is a character $\bmod N$ and $\psi_{l}$ is a character $\bmod p_{l}(l=1,2, \ldots, j)$.

Lemma 10. Let

$$
Q_{1, k}(n, \chi)=\sum_{\substack{\omega=1 \\(2 N, \omega)=1}}^{\infty} \chi(\omega) \omega^{-k} \sum_{r \bmod \omega} \exp (2 \pi i n r / \omega)
$$

Then $Q_{1, k}(n, \chi)=u^{1-k} \rho_{k-1}(u, \chi)$.

Proof. Since $(2 N, \omega)=1$, from (1.3) and (1.8) we have

$$
Q_{1, k}(n, \chi)=\sum_{\omega \mid u} \chi(\omega) \omega^{1-k}=u^{1-k} \sum_{\omega \mid u} \chi(\omega)\left(\frac{u}{\omega}\right)^{k-1}=u^{1-k} \rho_{k-1}(u, \chi) .
$$


The lemma is proved.

Lemma 11. Let $\chi(n)=\phi(n) \psi(n), M=p_{1}^{t_{1}} \cdots p_{j}^{t_{j}}$,

$$
Q_{2, k}(n, \chi)=\sum_{\alpha=0}^{\infty} 2^{-\alpha k} \psi^{\alpha}(2) \sum_{r \bmod 2^{\alpha+2}} \phi(r) \exp \left(2 \pi i n r / 2^{\alpha+2}\right) .
$$

Then

a) when $\phi=\phi_{0}, Q_{2, k}(n, \chi)=0$ if $2 \nmid n$ and

$$
Q_{2, k}(2 n, \chi)=2 \cdot 2^{s(1-k)} \psi^{s}(2)\left(2^{k-1} \bar{\psi}(2) \cdot \frac{2^{s(k-1)} \bar{\psi}^{s}(2)-1}{2^{k-1} \bar{\psi}(2)-1}-1\right) ;
$$

b) when $\phi \neq \phi_{0}, Q_{2, k}(n, \chi)=2 \cdot 2^{s(1-k)} \psi^{s}(2) \phi(M u) i$.

Proof. Any $r \in \mathbb{Z} / 2^{\alpha+2} \mathbb{Z}$ is written uniquely as $r=r_{1}+4 r_{2}, 0 \leq r_{1}<4$, $0 \leq r_{2}<2^{\alpha}$. Then $\phi(r)=\phi\left(r_{1}\right)$ and

$$
\begin{aligned}
\sum_{r \text { mod } 2^{\alpha+2}} \phi(r) & \exp \left(2 \pi i n r / 2^{\alpha+2}\right) \\
& =\sum_{r_{1} \bmod 4} \phi\left(r_{1}\right) \exp \left(2 \pi i n r_{1} / 2^{\alpha+2}\right) \sum_{r_{2} \bmod 2^{\alpha}} \exp \left(2 \pi i n r_{2} / 2^{\alpha}\right) .
\end{aligned}
$$

a) Let $\phi=\phi_{0}$. By virtue of (1.2), (1.3), from (2.2), (2.3) we get

1) if $2 \nmid n$, i.e., $s=0$, then

$$
\begin{aligned}
Q_{2, k}(n, \chi)= & \sum_{\alpha=0}^{\infty} 2^{-\alpha k} \psi^{\alpha}(2) \sum_{r_{1} \bmod 4} \phi_{0}\left(r_{1}\right) \exp \left(2 \pi i M u r_{1} / 2^{\alpha+2}\right) \\
& \times \sum_{r_{2} \bmod 2^{\alpha}} \exp \left(2 \pi i M u r_{2} / 2^{\alpha}\right)=\sum_{r_{1} \bmod 4} \phi_{0}\left(r_{1}\right) \exp \left(2 \pi i r_{1} / 4\right)=0 ;
\end{aligned}
$$

2) $Q_{2, k}(2 n, \chi)=\sum_{\alpha=0}^{\infty} 2^{-\alpha k} \psi^{\alpha}(2) \sum_{r_{1} \bmod 4} \phi_{0}\left(r_{1}\right) \exp \left(2 \pi i 2^{s+1} M_{u} r_{1} / 2^{\alpha+2}\right)$

$$
\begin{aligned}
& \times \sum_{r_{2} \bmod 2^{\alpha}} \exp \left(2 \pi i 2^{s+1} \text { Mur }_{2} / 2^{\alpha}\right) \\
= & \sum_{\alpha=0}^{s-1} 2 \cdot 2^{\alpha(1-k)} \psi^{\alpha}(2)-2 \cdot 2^{s(1-k)} \psi^{s}(2) \\
= & 2 \cdot \frac{1-2^{s(1-k)} \psi^{s}(2)}{1-2^{1-k} \psi(2)}-2 \cdot 2^{s(1-k)} \psi^{s}(2) \\
= & 2 \cdot 2^{s(1-k)} \psi^{s}(2)\left(2^{k-1} \bar{\psi}(2) \cdot \frac{2^{(k-1) s} \bar{\psi}^{s}(2)-1}{2^{k-1} \bar{\psi}(2)-1}-1\right) .
\end{aligned}
$$

b) Let $\phi \neq \phi_{0}$. Again using (1.2), (1.3), from (2.2), (2.3) we have

$$
Q_{2, k}(n, \chi)=2^{-s k} \psi^{s}(2) \cdot 2^{s} \sum_{r_{1} \bmod 4} \phi\left(r_{1}\right) \exp \left(2 \pi i M u r_{1} / 4\right)
$$




$$
=2^{(1-k) s} \psi^{s}(2) \cdot 2 i \phi(M u) \text {. }
$$

Lemma 12. Let $M_{l}=\prod_{\substack{l_{1}=1 \\ l_{1} \neq l}}^{j} p_{l_{1}}^{t_{l_{1}}}(1 \leq l \leq j), \chi(n)=\phi(n) \psi_{l}(n) \psi_{2, l}(n)$, where $\psi_{l}$ is a character $\bmod p_{l}$ and $\psi_{2, l}$ is a character $\bmod \frac{N}{p_{l}}$,

$$
Q_{3, k, l}(n, \bar{\chi})=\sum_{\beta=0}^{\infty} p_{l}^{-\beta k} \phi^{\beta}\left(p_{l}\right) \bar{\psi}_{2, l}^{\beta+1}\left(p_{l}\right) \sum_{r \bmod p_{l}^{\beta+1}} \bar{\psi}_{l}(r) \exp \left(2 \pi i n r / p_{l}^{\beta+1}\right) .
$$

In that case,

a) if $\overline{\psi_{l}}$ is the main character, then

$$
\begin{gathered}
Q_{3, k, l}(n, \bar{\chi})=p_{l}^{(1-k) t_{l}} \phi^{t_{l}}\left(p_{l}\right) \bar{\psi}_{2, l}^{t_{l}+1}\left(p_{l}\right) \\
\times\left(\left(p_{l}-1\right) \phi\left(p_{l}\right) \psi_{2, l}\left(p_{l}\right) p_{l}^{k-1} \cdot \frac{p_{l}^{(k-1) t_{l}} \phi^{t_{l}}\left(p_{l}\right) \psi_{2, l}^{t_{l}}\left(p_{l}\right)-1}{p_{l}^{k-1} \phi\left(p_{l}\right) \psi_{2, l}\left(p_{l}\right)-1}-1\right) ;
\end{gathered}
$$

b) if $\bar{\psi}_{l}$ is not the main character, then

$$
Q_{3, k, l}(n, \bar{\chi})=p_{l}^{(1-k) t_{l}} \phi^{t_{l}}\left(p_{l}\right) \bar{\psi}_{2, l}^{t_{l}+1}\left(p_{l}\right) \sqrt{p_{l}} \psi_{l}^{s}(2) \psi_{l}(M u) g\left(\bar{\psi}_{l}\right) .
$$

Proof. Any $r \in \mathbb{Z} / p_{l}^{\beta+1} \mathbb{Z}$ is written uniquely as $r=r_{1}+p_{l} r_{2}, 0 \leq r_{1}<p_{l}$, $0 \leq r_{2}<p_{l}^{\beta}$. Then $\bar{\psi}_{l}(r)=\bar{\psi}_{l}\left(r_{1}\right)$ and

$$
\begin{aligned}
& \sum_{r \bmod p_{l}^{\beta+1}} \bar{\psi}_{l}(r) \exp \left(2 \pi i n r / p_{l}^{\beta+1}\right) \\
& \quad=\sum_{r_{1} \bmod p_{l}} \bar{\psi}_{l}\left(r_{1}\right) \exp \left(2 \pi i n r_{1} / p_{l}^{\beta+1}\right) \sum_{r_{2} \bmod p_{l}^{\beta}} \exp \left(2 \pi i n r_{2} / p_{l}^{\beta}\right) .
\end{aligned}
$$

a) If $\bar{\psi}_{l}=\psi_{0}$, by virtue of $(1.1),(1.3)$, from (2.4), (2.5) we get

$$
\begin{aligned}
Q_{3, k, l}( & n, \bar{\chi})=\left(p_{l}-1\right) \sum_{\beta=0}^{t_{l}-1} p_{l}^{\beta(1-k)} \phi^{\beta}\left(p_{l}\right) \bar{\psi}_{2, l}^{\beta+1}\left(p_{l}\right)-p_{l}^{t_{l}(1-k)} \phi^{t_{l}}\left(p_{l}\right) \bar{\psi}_{2, l}^{t_{l}+1}\left(p_{l}\right) \\
& =\bar{\psi}_{2, l}\left(p_{l}\right)\left(\left(p_{l}-1\right) \cdot \frac{1-p_{l}^{t_{l}(1-k)} \phi^{t_{l}}\left(p_{l}\right) \bar{\psi}_{2, l}^{t_{l}}\left(p_{l}\right)}{1-p_{l}^{1-k} \phi\left(p_{l}\right) \bar{\psi}_{2, l}\left(p_{l}\right)}-p_{l}^{t_{l}(1-k)} \phi^{t_{l}}\left(p_{l}\right) \bar{\psi}_{2, l}^{t_{l}}\left(p_{l}\right)\right) \\
& =p_{l}^{(1-k) t_{l}} \phi^{t_{l}}\left(p_{l}\right) \bar{\psi}_{2, l}^{t_{l}+1}\left(p_{l}\right) \\
& \times\left(\left(p_{l}-1\right) \phi\left(p_{l}\right) \psi_{2, l}\left(p_{l}\right) p_{l}^{k-1} \cdot \frac{p_{l}^{(k-1) t_{l}} \phi^{t_{l}}\left(p_{l}\right) \psi_{2, l}^{t_{l}}\left(p_{l}\right)-1}{p_{l}^{k-1} \phi\left(p_{l}\right) \psi_{2, l}\left(p_{l}\right)-1}-1\right) .
\end{aligned}
$$

b) If $\bar{\psi}_{l} \neq \psi_{0}$, using (1.1), (1.3), from (2.4), (2.5) we have

$$
\begin{aligned}
Q_{3, k, l}(n, \bar{\chi}) & =p_{l}^{-t_{l} k} \phi^{t_{l}}\left(p_{l}\right) \bar{\psi}_{2, l}^{t_{l}+1}\left(p_{l}\right) p_{l}^{t_{l}} \sum_{r_{1} \bmod p_{l}} \bar{\psi}_{l}\left(r_{1}\right) \exp \left(2 \pi i 2^{s} M_{l} u r_{1} / p_{l}\right) \\
& =p_{l}^{(1-k) t_{l}} \phi^{t_{l}}\left(p_{l}\right) \bar{\psi}_{2, l}^{t_{l}+1}\left(p_{l}\right) \sqrt{p_{l}} \psi_{l}\left(2^{s} M_{l} u\right) g\left(\bar{\psi}_{l}\right) .
\end{aligned}
$$


Proposition 1. Let $\chi(n)=\phi(n) \psi_{1, l}(n) \psi_{2, l}(n)$, where $\psi_{1, l}$ is a character $\bmod N_{l}\left(N_{l} \mid N\right)$ and $\psi_{2, l}$ is a character $\bmod \left(N / N_{l}\right) ; \chi(-1)=(-1)^{k}$. Then

a) if $\phi=\phi_{0}$, then the system of the functions

$$
\begin{aligned}
E_{1}(\tau ; k, 4 N, \chi) & =\sum_{\substack{4 N \mid m \\
n>0,(m, n)=1}} \bar{\chi}(n)(m \tau+n)^{-k}, \\
E_{2, l}(\tau ; k, 4 N, \chi)= & \sum_{\substack{m>0, n \\
\left(2 N_{l} m, N n / N_{l}\right)=1}} \phi(n) \bar{\psi}_{1, l}(n) \psi_{2, l}(m)\left(4 N_{l} m \tau+n\right)^{-k}
\end{aligned}
$$

$\left(N_{l} \mid N, N_{l} \neq N, \quad l=1, \ldots, 2^{j}-1, \quad N_{l_{1}} \neq N_{l_{2}}\right.$, when $\left.l_{1} \neq l_{2}\right)$,

$$
E_{3, l}(\tau ; k, 4 N, \chi)=\sum_{\substack{m>0, n \\\left(N_{l} m, 2 n N / N_{l}\right)=1}} \phi(m) \psi_{2, l}(m) \bar{\psi}_{1, l}(n)\left(N_{l} m \tau+n\right)^{-k}
$$

$$
\left(N_{l} \mid N, \quad l=1, \ldots, 2^{j}, \quad N_{l_{1}} \neq N_{l_{2}}, \text { when } l_{1} \neq l_{2}\right),
$$

$$
E_{4, l}(\tau ; k, 4 N, \chi)=\sum_{\substack{2 \nmid m, m>0 \\ n,\left(2 N_{l} m, n N / N_{l}\right)=1}} \phi(n) \psi_{2, l}(m) \bar{\psi}_{1, l}(n)\left(2 N_{l} m \tau+n\right)^{-k}
$$

$$
\left(N_{l} \mid N, \quad l=1, \ldots, 2^{j}, \quad N_{l_{1}} \neq N_{l_{2}}, \text { when } l_{1} \neq l_{2}\right)
$$

is the basis of the space $E_{k}\left(\Gamma_{0}(4 N), \chi\right)$ for any $k \geq 3$;

b) if $\phi \neq \phi_{0}$, then the system of functions (2.6)-(2.8) is the basis of the space $E_{k}\left(\Gamma_{0}(4 N), \chi\right)$ for any $k \geq 3$.

Proof. It is well known (see [1], [5]) that to each regular cusp of the group $\Gamma_{0}(4 N)$ there corresponds an Eisenstein series and these functions form the basis of the space $E_{k}\left(\Gamma_{0}(4 N), \chi\right)$. Now the result follows from Lemmas 6 and 8 after easy calculations.

Next, we derive Fourier expansions of functions (2.6)-(2.9).

Let $a_{k}(\chi)=\frac{\pi^{k}}{i^{k} \Gamma(k) \mathcal{L}(k, \chi)}, \chi_{1}(n)=\phi(n) \psi_{2, l}(n) \bar{\psi}_{1, l}(n), \chi_{2}(n)=\phi_{0}(n) \psi_{2, l}(n) \bar{\psi}_{1, l}(n)$.

1) Since $\bar{\chi}(-1)=(-1)^{k}$, we have

$$
\sum_{\substack{m<0 \\ 4 N \mid m, n>0 \\(m, n)=1}} \bar{\chi}(n)(m \tau+n)^{-k}=\sum_{\substack{m>0 \\ 4 N \mid m, n>0 \\(m, n)=1}} \bar{\chi}(n)(-m \tau+n)^{-k}=\sum_{\substack{m>0 \\ 4 N \mid m, n<0 \\(m, n)=1}} \bar{\chi}(n)(m \tau+n)^{-k} .
$$

Thus, because of the absolute convergence, we have

$$
\begin{aligned}
E_{1}(\tau ; k, 4 N, \chi) & =1+\sum_{\substack{m>0 \\
n,(m, n)=1}} \bar{\chi}(n)(4 N m \tau+n)^{-k} \\
& =1+\left(\sum_{d=1}^{\infty} \bar{\chi}(d) d^{-k}\right)^{-1} \sum_{\substack{m>0 \\
n}} \bar{\chi}(n)(4 N m \tau+n)^{-k}
\end{aligned}
$$


Let $n=r+4 N m h, r \bmod 4 N m, h \in \mathbb{Z}$. Then $\bar{\chi}(n)=\bar{\chi}(r)=\phi(r) \bar{\psi}(r)$. By Lemma 5 and (1.9) we get

$$
\begin{aligned}
& E_{1}(\tau ; k, 4 N, \chi)=1+(4 N)^{-k} \mathcal{L}(k, \bar{\chi})^{-1} \\
& \quad \times \sum_{m=1}^{\infty}\left(m^{-k} \sum_{r \bmod 4 N m} \bar{\chi}(r) \sum_{h=-\infty}^{\infty}\left(\tau+\frac{r}{4 N m}+h\right)^{-k}\right) \\
& =1+(4 N)^{-k}(2 \pi)^{k} \exp (-\pi i k / 2)(\Gamma(k) \mathcal{L}(k, \bar{\chi}))^{-1} \\
& \quad \times \sum_{n=1}^{\infty}\left(n^{k-1} \sum_{m=1}^{\infty} m^{-k} \sum_{r \bmod 4 N m} \bar{\chi}(r) \exp (2 \pi i n r /(4 N m))\right) z^{n} \\
& =1+(2 N)^{-k} a_{k}(\bar{\chi}) \sum_{n=1}^{\infty}\left(n^{k-1} \sum_{\alpha=0}^{\infty} \sum_{\beta_{1}=0}^{\infty} \cdots \sum_{\beta_{j}=0}^{\infty} \sum_{(2 N=1}^{\infty}\left(2^{\alpha} p_{1}^{\beta_{1}} \cdots p_{j}^{\beta_{j}} \omega\right)^{-k}\right. \\
& \quad \times \quad \sum_{\left.(r) \bar{\psi}(r) \exp \left(2 \pi i n r /\left(2^{\alpha+2} p_{1}^{\beta_{1}+1} \cdots p_{j}^{\beta_{j}+1} \omega\right)\right)\right) z^{n} .} \\
& \quad r \bmod 2^{\alpha+2} p_{1}^{\beta_{1}+1} \ldots p_{j}^{\beta_{j}+1} \omega
\end{aligned}
$$

Any $r \in \mathbb{Z} / 2^{\alpha+2} p_{1}^{\beta_{1}+1} \cdots p_{j}^{\beta_{j}+1} \omega \mathbb{Z}$ is written uniquely as

$$
\begin{gathered}
r=\omega \cdot 2^{\alpha+2} \sum_{h=1}^{j} r_{h} \prod_{\substack{h_{1}=1 \\
h_{1} \neq h}}^{j} p_{h_{1}}^{\beta_{h_{1}}+1}+\omega r_{j+1} \prod_{h=1}^{j} p_{h}^{\beta_{h}+1}+2^{\alpha+2} r_{j+2} \prod_{h=1}^{j} p_{h}^{\beta_{h}+1}, \\
0 \leq r_{j+2}<\omega, \quad 0 \leq r_{h}<p_{h}^{\beta_{h}+1}, \quad 1 \leq h \leq j, \quad 0 \leq r_{j+1}<2^{\alpha+2} .
\end{gathered}
$$

Then

$$
\begin{gathered}
\phi(r)=\phi\left(r_{j+1}\right) \phi(\omega) \prod_{l=1}^{j} \phi\left(p_{l}^{\beta_{l}+1}\right) \\
\bar{\psi}_{l}(r)=\bar{\psi}_{l}(\omega) \bar{\psi}_{l}(4) \bar{\psi}_{l}^{\alpha}(2) \bar{\psi}_{l}\left(r_{l}\right) \prod_{\substack{l_{1}=1 \\
l_{1} \neq l}}^{j} \bar{\psi}_{l}\left(p_{l_{1}}^{\beta_{l}+1}\right), \quad \phi(\omega) \prod_{l=1}^{j} \bar{\psi}_{l}(\omega)=\bar{\chi}(\omega), \\
\prod_{l=1}^{j} \bar{\psi}_{l}\left(2^{\alpha}\right)=\bar{\psi}^{\alpha}(2), \quad \prod_{l=1}^{j} \bar{\psi}_{l}(4)=\bar{\psi}(4) \\
\exp \left(2 \pi i n r /\left(2^{\alpha+2} p_{1}^{\beta_{1}+1} \cdots p_{j}^{\beta_{j}+1} \omega\right)\right)=\exp \left(2 \pi i n r_{j+2} / \omega\right) \\
\times \prod_{l=1}^{j} \exp \left(2 \pi i n r_{l} / p_{l}^{\beta_{l}+1}\right) \exp \left(2 \pi i n r_{j+1} / 2^{\alpha+2}\right)
\end{gathered}
$$

and from (2.10) we obtain

$$
E_{1}(\tau ; k, 4 N, \chi)=1+(2 N)^{-k} a_{k}(\bar{\chi}) \bar{\psi}(4) \phi(N)
$$


ON THE FOURIER EXPANSIONS OF EISENSTEIN SERIES OF SOME TYPES

$$
\begin{aligned}
& \times \sum_{n=1}^{\infty}\left(n^{k-1} \sum_{\substack{\omega=1 \\
(2 N, \omega)=1}}^{\infty} \bar{\chi}(\omega) \omega^{-k} \sum_{r_{j+2} \bmod \omega} \exp \left(2 \pi i n r_{j+2} / \omega\right)\right. \\
& \times \sum_{\alpha=0}^{\infty} 2^{-\alpha k} \bar{\psi}^{\alpha}(2) \sum_{r_{j+1} \bmod 2^{\alpha+2}} \phi\left(r_{j+1}\right) \exp \left(2 \pi i n r_{j+1} / 2^{\alpha+2}\right) \\
& \times \prod_{l=1}^{j}\left(\sum_{\beta_{l}=0}^{\infty} p_{l}^{-\beta_{l} k} \phi^{\beta_{l}}\left(p_{l}\right) \prod_{l_{1}=1}^{j} \bar{\psi}_{l_{1}}\left(p_{l}^{\beta_{l}+1}\right)\right. \\
& \left.\left.\times \sum_{l_{1} \neq l} \bar{\psi}_{l}\left(r_{l}\right) \exp \left(2 \pi i n r_{l} / p_{l}^{\beta_{l}+1}\right)\right)\right) z^{n} \\
& =1+a_{k}(\bar{\chi}) \bar{\psi}_{(4) \phi(N) 2^{-k} N^{-k}}(4.11) \\
& \times \sum_{n=1}^{\infty} n^{k-1} Q_{1, k}(n, \bar{\chi}) Q_{2, k}(n, \bar{\chi}) \prod_{l=1}^{j} Q_{3, k, l}(n, \bar{\chi}) z^{n} .
\end{aligned}
$$

2) $E_{2, l}(\tau ; k, 4 N, \chi)=\sum_{\substack{m>0, n \\(m, n)=1}} \phi(n) \bar{\psi}_{1, l}(n) \psi_{2, l}(m)\left(4 N_{l} m \tau+n\right)^{-k}$

$$
=\frac{1}{\mathcal{L}\left(k, \chi_{1}\right)} \sum_{m=1}^{\infty} \psi_{2, l}(m) \sum_{n=-\infty}^{\infty} \phi(n) \bar{\psi}_{1, l}(n)\left(4 N_{l} m \tau+n\right)^{-k} .
$$

Let $n=r+4 N_{l} m h, r \bmod 4 N_{l} m, h \in \mathbb{Z}$. Then $\phi(n)=\phi(r), \bar{\psi}_{1, l}(n)=\bar{\psi}_{1, l}(r)$, and by Lemma 5 we get

$$
\begin{aligned}
& E_{2, l}(\tau ; k, 4 N, \chi)=\left(4 N_{l}\right)^{-k} \mathcal{L}\left(k, \chi_{1}\right)^{-1} \\
& \quad \times \sum_{m=1}^{\infty}\left(\psi_{2, l}(m) m^{-k} \sum_{r \bmod 4 N_{l} m} \phi(r) \bar{\psi}_{1, l}(r) \sum_{h=-\infty}^{\infty}\left(\tau+\frac{r}{4 N_{l} m}+h\right)^{-k}\right) \\
& =\left(2 N_{l}\right)^{-k} a_{k}\left(\chi_{1}\right) \sum_{n=1}^{\infty}\left(n^{k-1} \sum_{\substack{m=1 \\
\left(N / N_{l}, m\right)=1}}^{\infty} \psi_{2, l}(m) m^{-k}\right. \\
& \left.\quad \times \sum_{r \bmod 4 N_{l} m} \phi(r) \bar{\psi}_{1, l}(r) \exp \left(2 \pi i n r /\left(4 N_{l} m\right)\right)\right) z^{n} .
\end{aligned}
$$

Let $N_{l}=p_{\delta_{1}} \cdots p_{\delta_{d}}, 1 \leq d \leq j-1$, or $N_{l}=1$, then

$$
\begin{aligned}
& E_{2, l}(\tau ; k, 4 N, \chi)=\left(2 N_{l}\right)^{-k} a_{k}\left(\chi_{1}\right) \\
& \quad \times \sum_{n=1}^{\infty}\left(n^{k-1} \sum_{\alpha=0}^{\infty} \sum_{\beta_{1}=0}^{\infty} \cdots \sum_{\beta_{d}=0}^{\infty} \sum_{\substack{\omega=1 \\
(N, \omega)=1}}^{\infty} \psi_{2, l}\left(2^{\alpha} p_{\delta_{1}}^{\beta_{1}} \cdots p_{\delta_{d}}^{\beta_{d}} \omega\right)\left(2^{\alpha} p_{\delta_{1}}^{\beta_{1}} \cdots p_{\delta_{d}}^{\beta_{d}} \omega\right)^{-k}\right.
\end{aligned}
$$




$$
\left.\times \sum_{r \bmod 2^{\alpha+2} p_{\delta_{1}}^{\beta_{1}+1} \cdots p_{\delta_{d}}^{\beta_{d}+1} \omega} \phi(r) \bar{\psi}_{1, l}(r) \exp \left(2 \pi i n r /\left(2^{\alpha+2} p_{\delta_{1}}^{\beta_{1}+1} \cdots p_{\delta_{d}}^{\beta_{d}+1} \omega\right)\right)\right) z^{n} .
$$

Let

$$
\begin{gathered}
r=\omega \cdot 2^{\alpha+2} \sum_{h=1}^{d} r_{h} \prod_{\substack{h_{1}=1 \\
h_{1} \neq h}}^{d} p_{\delta_{h_{1}}}^{\beta_{h_{1}+1}}+\omega r_{d+1} \prod_{h=1}^{d} p_{\delta_{h}}^{\beta_{h}+1}+2^{\alpha+2} r_{d+2} \prod_{h=1}^{d} p_{\delta_{h}}^{\beta_{h}+1}, \\
0 \leq r_{d+2}<\omega, \quad 0 \leq r_{h}<p_{\delta_{h}}^{\beta_{h}+1}, \quad 1 \leq h \leq d, \quad 0 \leq r_{d+1}<2^{\alpha+2}
\end{gathered}
$$

Then

$$
\begin{aligned}
\phi(r) & =\phi\left(r_{d+1}\right) \phi(\omega) \phi\left(p_{\delta_{1}}^{\beta_{1}+1} \cdots p_{\delta_{d}}^{\beta_{d}+1}\right) \\
\bar{\psi}_{1, l}(r)=\prod_{h=1}^{d} \bar{\psi}_{\delta_{h}}(r) & =\prod_{h=1}^{d}\left(\bar{\psi}_{\delta_{h}}(\omega) \bar{\psi}_{\delta_{h}}\left(r_{h}\right) \bar{\psi}_{\delta_{h}}\left(2^{\alpha+2}\right) \prod_{\substack{h_{1}=1 \\
h_{1} \neq h}}^{d} \bar{\psi}_{\delta_{h}}\left(p_{\delta_{h_{1}}}^{\beta_{h_{1}}+1}\right)\right)
\end{aligned}
$$

and

$$
\begin{aligned}
E_{2, l}( & ; k, 4 N, \chi)=\left(2 N_{l}\right)^{-k} \bar{\psi}_{1, l}(4) \phi\left(N_{l}\right) \bar{\psi}_{2, l}\left(N_{l}\right) a_{k}\left(\chi_{1}\right) \\
\times & \sum_{n=1}^{\infty}\left(n^{k-1} \sum_{\substack{\omega=1 \\
(2 N, \omega)=1}}^{\infty} \chi_{1}(\omega) \omega^{-k} \sum_{r_{d+2} \bmod \omega} \exp \left(2 \pi i n r_{d+2} / \omega\right)\right. \\
& \times \sum_{\alpha=0}^{\infty} 2^{-\alpha k} \bar{\psi}_{1, l}^{\alpha}(2) \psi_{2, l}^{\alpha}(2) \sum_{r_{d+1} \bmod 2^{\alpha+2}} \phi\left(r_{d+1}\right) \exp \left(2 \pi i n r_{d+1} / 2^{\alpha+2}\right) \\
& \times \prod_{h=1}^{d}\left(\sum_{\beta_{h}=1}^{\infty} p_{\delta_{h}}^{-\beta_{h} k} \phi^{\beta_{h}}\left(p_{\delta_{h}}\right) \psi_{2, l}\left(p_{\delta_{h}}^{\beta_{h}+1}\right) \prod_{h_{1}=1}^{d} \bar{\psi}_{\delta_{h_{1}}}\left(p_{\delta_{h}}^{\beta_{h}+1}\right)\right. \\
& \left.\left.\times \sum_{h_{1} \neq h} \bar{\psi}_{\delta_{h}}\left(r_{h}\right) \exp \left(2 \pi i n r_{h} / p_{\delta_{h}}^{\beta_{h}+1}\right)\right)\right) z^{n} \\
= & \left(2 N_{l}\right)^{-k} \bar{\psi}_{1, l}(4) \phi\left(N_{l}\right) \bar{\psi}_{2, l}\left(N_{l}\right) a_{k}\left(\chi_{1}\right) \\
\times & \sum_{n=1}^{\infty}\left(n^{k-1} Q_{1, k}\left(n, \chi_{1}\right) Q_{2, k}\left(n, \chi_{1}\right) \prod_{h=1}^{d} Q_{3, k, \delta_{h}}\left(n, \chi_{1}\right)\right) z^{n} .
\end{aligned}
$$

3) If $N_{l}=1, d=0$ or $N_{l}=p_{\delta_{1}} \cdots p_{\delta_{d}}, 1 \leq d \leq j$, then similarly to the above we obtain

$$
\begin{aligned}
& E_{3, l}(\tau ; k, 4 N, \chi)=2^{k} N_{l}^{-k} a_{k}\left(\chi_{1}\right) \bar{\psi}_{2, l}\left(N_{l}\right) \sum_{n=1}^{\infty}\left(n^{k-1} Q_{1, k}\left(n, \chi_{1}\right)\right. \\
& \left.\times \prod_{h=1}^{d} Q_{3, k, \delta_{h}}\left(n, \chi_{1}\right)\right) z^{n}
\end{aligned}
$$


and

$$
\begin{aligned}
& E_{4, l}(\tau ; k, 4 N, \chi)=N_{l}^{-k} a_{k}\left(\chi_{2}\right) \bar{\psi}_{1, l}(2) \bar{\psi}_{2, l}\left(N_{l}\right) \sum_{n=1}^{\infty}\left(n^{k-1}(-1)^{n} Q_{1, k}\left(n, \chi_{2}\right)\right. \\
& \left.\quad \times \prod_{h=1}^{d} Q_{3, k, \delta_{h}}\left(n, \chi_{2}\right)\right) z^{n} .
\end{aligned}
$$

For $N=p$, we obtain the following results.

Corollary 1. Let $\chi(n)=\phi_{0}(n) \psi(n), \chi(-1)=(-1)^{k}, \psi \neq \psi_{0}, n=2^{s} p^{t} u$, $(2 p, u)=1, b_{k}(\chi)=\psi(2) 2^{k-1} \cdot \frac{\psi^{s}(2) \cdot 2^{(k-1) s}-1}{\psi(2) 2^{k-1}-1}-1$. Then

$$
\begin{aligned}
& E_{1}(\tau ; k, 4 p, \chi)=1+2^{1-k} a_{k}(\bar{\chi}) \bar{\psi}(2) g(\bar{\psi}) p^{\frac{1}{2}-k} \sum_{n=1}^{\infty} b_{k}(\chi) \psi(u) \rho_{k-1}(u, \bar{\chi}) z^{2 n} \\
& E_{2}(\tau ; k, 4 p, \chi)=2^{1-k} a_{k}(\chi) \sum_{n=1}^{\infty} b_{k}(\bar{\chi}) \psi^{s}(2) p^{(k-1) t} \rho_{k-1}(u, \chi) z^{2 n} \\
& E_{3,1}(\tau ; k, 4 p, \chi)=2^{k} a_{k}(\chi) \sum_{n=1}^{\infty} 2^{(k-1) s} p^{(k-1) t} \rho_{k-1}(u, \chi) z^{n}, \\
& E_{3,2}(\tau ; k, 4 p, \chi)=a_{k}(\bar{\chi}) g(\bar{\psi}) 2^{k} \cdot p^{\frac{1}{2}-k} \sum_{n=1}^{\infty} \psi^{s}(2) \psi(u) 2^{(k-1) s} \rho_{k-1}(u, \bar{\chi}) z^{n} \\
& E_{4,1}(\tau ; k, 4 p, \chi)=a_{k}(\chi) \sum_{n=1}^{\infty}(-1)^{n} \cdot 2^{(k-1) s} p^{(k-1) t} \rho_{k-1}(u, \chi) z^{n} \\
& E_{4,2}(\tau ; k, 4 p, \chi)=a_{k}(\bar{\chi}) \bar{\psi}(2) g(\bar{\psi}) p^{\frac{1}{2}-k} \sum_{n=1}^{\infty}(-1)^{n} \psi^{s}(2) \psi(u) 2^{(k-1) s} \rho_{k-1}(u, \bar{\chi}) z^{n} .
\end{aligned}
$$

Proof directly follows from (2.11)-(2.14) and Lemmas 10-12, replacing, in Lemma 11, $n$ and $s$ by $2 n$ and $s+1$ to obtain Fourier expansions for (2.6) and (2.7); furthermore, $\phi(p)=1$.

Corollary 2. Let $\chi(n)=\phi_{0}(n) \psi_{0}(n), n=2^{s} p^{t} u,(2 p, u)=1, b_{k}=2^{2 k-1}$. $\frac{2^{(2 k-1) s}-1}{2^{2 k-1}-1}-1, c_{k}=(p-1) p^{2 k-1} \cdot \frac{p^{(2 k-1) t}-1}{p^{2 k-1}-1}-1, d_{k}=\frac{(-1)^{k} 4 k(2 p)^{2 k}}{\left(2^{2 k}-1\right)\left(p^{2 k}-1\right) B_{k}}$, where $B_{k}$ are Bernoulli numbers. Then

$$
\begin{gathered}
E_{1}(\tau ; 2 k, 4 p, \chi)=1+(2 p)^{-2 k} d_{k} \sum_{n=1}^{\infty} b_{k} c_{k} \sigma_{2 k-1}(u) z^{2 n} \\
E_{2}(\tau ; 2 k, 4 p, \chi)=2^{-2 k} d_{k} \sum_{n=1}^{\infty} b_{k} p^{(2 k-1) t} \sigma_{2 k-1}(u) z^{2 n} \\
E_{3,1}(\tau ; 2 k, 4 p, \chi)=d_{k} \sum_{n=1}^{\infty} 2^{(2 k-1) s} p^{(2 k-1) t} \sigma_{2 k-1}(u) z^{n}
\end{gathered}
$$




$$
\begin{aligned}
& E_{3,2}(\tau ; 2 k, 4 p, \chi)=p^{-2 k} d_{k} \sum_{n=1}^{\infty} c_{k} \cdot 2^{(2 k-1) s} \sigma_{2 k-1}(u) z^{n} \\
& E_{4,1}(\tau ; 2 k, 4 p, \chi)=2^{-2 k} d_{k} \sum_{n=1}^{\infty}(-1)^{n} 2^{(2 k-1) s} p^{(2 k-1) t} \sigma_{2 k-1}(u) z^{n} \\
& E_{4,2}(\tau ; 2 k, 4 p, \chi)=(2 p)^{-2 k} d_{k} \sum_{n=1}^{\infty}(-1)^{n} c_{k} 2^{(2 k-1) s} \sigma_{2 k-1}(u) z^{n}
\end{aligned}
$$

Proof. It follows from (1.9) and (1.11) that $\mathcal{L}\left(2 k, \chi_{0}\right)=\left(1-2^{-2 k}\right)\left(1-p^{-2 k}\right) \zeta(2 k)$. It is known (see [7], Ch. I) that $\zeta(2 k)=\frac{2^{2 k-1} \pi^{2 k}}{(2 k) !} B_{k}$. Then the result immediately follows from (2.11)-(2.14) and Lemmas 10-12.

Corollary 3. Let $\chi(n)=\phi(n) \psi(n), \phi \neq \phi_{0}, \psi \neq \psi_{0}, n=2^{s} p^{t} u,(2 p, u)=1$; then

$$
\begin{aligned}
E_{1}(\tau ; k, 4 p, \chi) & =1+i \cdot 2^{1-k} p^{\frac{1}{2}-k} a_{k}(\bar{\chi}) \bar{\psi}(4) \phi(p) g(\bar{\psi}) \sum_{n=1}^{\infty} \chi(u) \rho_{k-1}(u, \bar{\chi}) z^{n}, \\
E_{2}(\tau ; k, 4 p, \chi) & =2^{1-k} i a_{k}(\chi) \sum_{n=1}^{\infty} p^{(k-1) t} \psi^{s}(2) \phi^{t}(p) \phi(u) \rho_{k-1}(u, \chi) z^{n}, \\
E_{3,1}(\tau ; k, 4 p, \chi) & =2^{k} \cdot a_{k}(\chi) \sum_{n=1}^{\infty} 2^{(k-1) s} p^{(k-1) t} \rho_{k-1}(u, \chi) z^{n} \\
E_{3,2}(\tau ; k, 4 p, \chi) & =2^{k} \cdot p^{\frac{1}{2}-k} a_{k}(\bar{\chi}) g(\bar{\psi}) \sum_{n=1}^{\infty} 2^{(k-1) s} \phi^{t}(p) \psi^{s}(2) \psi(u) \rho_{k-1}(u, \bar{\chi}) z^{n} .
\end{aligned}
$$

Proof directly follows from (2.11)-(2.13) and Lemmas 10-12.

Corollary 4. Let $\chi(n)=\phi(n) \psi_{0}(n), \phi \neq \phi_{0}, n=2^{s} p^{t} u,(2 p, u)=1$, $c_{k}=\phi(p)(p-1) p^{2 k} \cdot \frac{\phi^{t}(p) p^{2 k t}-1}{\phi(p) p^{2 k}-1}-1, d_{k}=\frac{4(-1)^{k}}{\left(1-\phi(p) p^{-(2 k+1)}\right) E_{k}}$, where $E_{k}$ are Euler numbers. Then

$$
\begin{aligned}
E_{1}(\tau ; 2 k+1,4 p, \chi) & =1+\phi(p) p^{-(2 k+1)} d_{k} \sum_{n=1}^{\infty} c_{k} \phi(u) \rho_{2 k}(u, \chi) z^{n}, \\
E_{2}(\tau ; 2 k+1,4 p, \chi) & =d_{k} \sum_{n=1}^{\infty} \phi^{t}(p) \phi(u) p^{2 k t} \rho_{2 k}(u, \chi) z^{n} \\
E_{3,1}(\tau ; 2 k+1,4 p, \chi) & =-i \cdot 2^{4 k+1} d_{k} \sum_{n=1}^{\infty} 2^{2 k s} p^{2 k t} \rho_{2 k}(u, \chi) z^{n} \\
E_{3,2}(\tau ; 2 k+1,4 p, \chi) & =-i \cdot 2^{4 k+1} p^{-(2 k+1)} d_{k} \sum_{n=1}^{\infty} c_{k} \phi^{t}(p) 2^{2 k s} \rho_{2 k}(u, \chi) z^{n} .
\end{aligned}
$$

Proof. It follows from (1.9) that

$$
\mathcal{L}(2 k+1, \chi)=\sum_{\substack{n=1 \\ p \nmid n}}^{\infty}(-1)^{n-1}(2 n-1)^{-(2 k+1)}
$$




$$
\begin{aligned}
& =\left(1-\phi(p) p^{-(2 k+1)}\right) \sum_{n=1}^{\infty}(-1)^{n-1}(2 n-1)^{-(2 k+1)} \\
& =\left(1-\phi(p) p^{-(2 k+1)}\right) \cdot \frac{\pi^{2 k+1}}{2^{2 k+2}(2 k) !} E_{k} \quad(\text { see [7], Ch. I). }
\end{aligned}
$$

Then the result follows from (2.11)-(2.13) and Lemmas 10-12.

3. In what follows, let $n=2^{2 s} p_{1}^{2 t_{1}} \cdots p_{j}^{2 t_{j}} n_{1}^{2} u, N=p_{1} \cdots p_{j},\left(2 N, n_{1}\right)=1$, where $u$ is square-free, $2 \nmid k, M=p_{1}^{2 t_{1}} \cdots p_{j}^{2 t_{j}}, \chi=\phi \psi$ is a character $\bmod 4 N$.

Lemma 13. Let

$$
S_{1, k}(n, \chi)=\sum_{\alpha=0}^{\infty}\left(2^{-\frac{k}{2} \alpha} \psi^{\alpha}(2) \sum_{\substack{r+\bmod _{2} 2^{\alpha+2} \\ 2 \nmid r}} \phi(r)\left(\frac{2}{r}\right)^{\alpha} \varepsilon_{r}^{k} \exp \left(2 \pi i n r / 2^{\alpha+2}\right)\right) \text {. }
$$

Then

$$
\begin{aligned}
S_{1, k}(n, \chi)= & 2^{(2-k) s} \psi^{s}(4)\left(1+\phi(3)\left(\frac{-1}{k}\right) i\right) \\
& \times\left(2^{k-2} \bar{\psi}(4) \cdot \frac{2^{(k-2) s} \bar{\psi}^{s}(4)-1}{2^{k-2} \bar{\psi}(4)-1}+c_{k}(u, \chi)\right),
\end{aligned}
$$

where

$$
c_{k}(u, \chi)= \begin{cases}-1 & \text { if }(-1)^{\frac{k-1}{2}} \phi(3) u \neq 1(\bmod 4) ; \\ 1+2^{\frac{3-k}{2}} \psi(2)\left(\frac{2}{u}\right) & \text { if }(-1)^{\frac{k-1}{2}} \phi(3) u \equiv 1(\bmod 4) .\end{cases}
$$

Proof. It is well-known that $\left(\frac{2}{r}\right)=(-1)^{\left(r^{2}-1\right) / 8}$, therefore $\left(\frac{2}{4 r+1}\right)=(-1)^{r}$ and $\left(\frac{2}{4 r+3}\right)=(-1)^{r+1}$; furthermore, $\varepsilon_{r}=1, \phi(r)=1$ when $r \equiv 1(\bmod 4)$, and $\varepsilon_{r}=i, \phi(r)=\phi(3)$ when $r \equiv 3(\bmod 4)$. Hence

$$
\begin{aligned}
S_{1, k}(n, \chi) & =\sum_{\alpha=0}^{\infty}\left(2^{-\frac{k}{2} \alpha} \psi^{\alpha}(2) \exp \left(2 \pi i n / 2^{\alpha+2}\right)\right. \\
& \left.\times\left(1+(-1)^{\alpha} \phi(3) i^{k} \exp \left(2 \pi i n / 2^{\alpha+1}\right)\right) \sum_{r \bmod 2^{\alpha}}(-1)^{r \alpha} \exp \left(2 \pi i n r / 2^{\alpha}\right)\right) \\
= & \exp (2 \pi i n / 4)\left(1+\phi(3) i^{k} \exp (\pi i n)\right) \\
& +\sum_{\alpha=1}^{\infty}\left(2^{-\frac{k}{2} \alpha} \psi^{\alpha}(2) \exp \left(2 \pi i n / 2^{\alpha+2}\right)\left(1+(-1)^{\alpha} \phi(3) i^{k} \exp \left(2 \pi i n / 2^{\alpha+1}\right)\right)\right. \\
& \left.\times\left(1+(-1)^{\alpha} \exp \left(2 \pi i n / 2^{\alpha}\right)\right) \sum_{r \bmod 2^{\alpha-1}} \exp \left(2 \pi i n r / 2^{\alpha-1}\right)\right) .
\end{aligned}
$$

Since $2 \nmid n_{1}$, we have $M n_{1}^{2} \equiv 1(\bmod 8)$ and

$$
\exp \left(2 \pi i n / 2^{2 s+3}\right)=\exp (\pi i u / 4)=\frac{1}{\sqrt{2}} \cdot\left(\frac{2}{u}\right)\left(1+\left(\frac{-1}{u}\right) i\right) \text { when } 2 \nmid u ;
$$




$$
\exp \left(2 \pi i n / 2^{2 s+1}\right)=(-1)^{u}, \quad \exp \left(2 \pi i n / 2^{2 s+2}\right)=i^{u}
$$

if $2 \nmid u$, then $i^{u}=\left(\frac{-1}{u}\right) i ; 2 \nmid k$, hence $i^{k}=\left(\frac{-1}{k}\right) i$. It is easy to verify that

$$
\begin{aligned}
1+(-1)^{\alpha} \exp \left(2 \pi i n / 2^{\alpha}\right)= & 1+(-1)^{\alpha} \text { when } \alpha \leq 2 s ; \\
& 1-(-1)^{u} \text { when } \alpha=2 s+1 ; \\
& 0 \text { when } \alpha=2 s+2 \text { and } 2 \mid u .
\end{aligned}
$$

Now from (1.3) and (3.3) we get

$$
\begin{aligned}
S_{1, k}(n, \chi)= & \sum_{\alpha=0}^{s-1} 2^{(2-k) \alpha} \psi^{\alpha}(4)\left(1+\phi(3)\left(\frac{-1}{k}\right) i\right) \\
& +2^{(2-k) s} \psi^{s}(4) i^{u}\left(1+\phi(3)\left(\frac{-1}{k}\right) i \cdot(-1)^{u}\right) \\
& +2^{(2-k) s} \cdot 2^{-\frac{k}{2}} \psi^{s}(4) \psi(2) \exp (\pi i u / 4) \\
& \times\left(1-\phi(3)\left(\frac{-1}{k}\right) i \cdot i^{u}\right)\left(1-(-1)^{u}\right) .
\end{aligned}
$$

If $(-1)^{\frac{k-1}{2}} \phi(3) u \equiv 1(\bmod 4)$, then $\left(\frac{-1}{u}\right)=\phi(3)\left(\frac{-1}{k}\right)$, and if $(-1)^{\frac{k-1}{2}} \phi(3) u \equiv$ $3(\bmod 4)$, then $\left(\frac{-1}{u}\right)=-\phi(3)\left(\frac{-1}{k}\right)$. Therefore, using (3.4) and (3.5), we have

$$
\begin{aligned}
& i^{u}\left(1+\phi(3)\left(\frac{-1}{k}\right) i \cdot(-1)^{u}\right)+2^{-\frac{k}{2}} \psi(2) \exp (\pi i u / 4) \\
& \times\left(1-\phi(3)\left(\frac{-1}{k}\right) i \cdot i^{u}\right)\left(1-(-1)^{u}\right)=\left(1+\phi(3)\left(\frac{-1}{k}\right) i\right) c_{k}(u, \chi) .
\end{aligned}
$$

Furthermore,

$$
\begin{aligned}
\sum_{\alpha=0}^{s-1} 2^{(2-k) \alpha} \psi^{\alpha}(4) & =\frac{1-2^{(2-k) s} \psi^{s}(4)}{1-2^{2-k} \psi(4)} \\
& =2^{(2-k) s} \psi^{s}(4) 2^{k-2} \bar{\psi}(4) \cdot \frac{2^{(k-2) s} \bar{\psi}^{s}(4)-1}{2^{k-2} \bar{\psi}(4)-1}
\end{aligned}
$$

From (3.6)-(3.8) we obtain (3.2).

Lemma 14. Let $\psi_{l}(n)$ be a character $\bmod p_{l}, M_{l}=\prod_{l_{1}=1, l_{1} \neq l}^{j} p_{l_{1}}^{2 t_{l_{1}}}, 1 \leq l \leq j$,

$$
S_{2, k, l}\left(n, \psi_{l}\right)=\sum_{\substack{r \bmod _{l}^{\beta+1} \\ p_{l} \nmid r}} \psi_{l}(r)\left(\frac{r}{p_{l}}\right)^{\beta+1} \exp \left(2 \pi i n r / p_{l}^{\beta+1}\right), \quad(1 \leq l \leq j) .
$$

Then

a) if $\psi_{l}$ is the main character $\bmod p_{l}$, then

$$
\begin{aligned}
S_{2, k, l}\left(n, \psi_{l}\right)= & \left(p_{l}-1\right) p_{l}^{\beta} \quad \text { if } 2 \nmid \beta, 1 \leq \beta \leq 2 t_{l}-1, \\
& -p_{l}^{2 t_{l}+1} \text { if } \beta=2 t_{l}+1, p_{l} \mid u,
\end{aligned}
$$




$$
\left(\frac{u}{p_{l}}\right) \varepsilon_{p_{l}} p_{l}^{2 t_{l}} \sqrt{p_{l}} \text { if } \beta=2 t_{l}, p_{l} \nmid u,
$$

0 otherwise;

b) if $\psi_{l}=\left(\frac{\dot{\bar{p}}}{p_{l}}\right.$, then

$$
\begin{aligned}
S_{2, k, l}\left(n, \psi_{l}\right)= & \left(p_{l}-1\right) p_{l}^{\beta} \text { if } 2 \mid \beta, \beta \leq 2 t_{l}-2 \text { or } \beta=2 t_{l}, p_{l} \mid u, \\
& -p_{l}^{2 t_{l}} \text { if } \beta=2 t_{l}, p_{l} \nmid u, \\
& \left(\frac{u / p_{l}}{p_{l}}\right) \varepsilon_{p_{l}} p_{l}^{2 t_{l}+1} \sqrt{p_{l}} \text { if } \beta=2 t_{l}+1, p_{l} \mid u, \\
& 0 \text { otherwise; }
\end{aligned}
$$

c) if $\bar{\psi}_{l} \neq \psi_{l}$, then

$$
\begin{aligned}
S_{2, k, l}\left(n, \psi_{l}\right)= & \sqrt{p_{l}} p_{l}^{2 t_{l}} \bar{\psi}_{l}\left(4^{s} M_{l} n_{1}^{2} u\right)\left(\frac{u}{p_{l}}\right) g\left(\psi_{l}\left(\frac{\cdot}{p_{l}}\right)\right) \text { if } \beta=2 t_{l}, \quad p_{l} \nmid u, \\
& \sqrt{p_{l}} p_{l}^{2 t_{l}+1} \bar{\psi}_{l}\left(4^{s} M_{l} n_{1}^{2} u / p_{l}\right) g\left(\psi_{l}\right) \text { if } \beta=2 t_{l}+1, \quad p_{l} \mid u, \\
& 0 \text { otherwise. }
\end{aligned}
$$

Proof. Let $r=r_{1}+r_{2} p_{l}, r_{1} \bmod p_{l}, p_{l} \nmid r_{1}, r_{2} \bmod p_{l}^{\beta}$. Then $\psi_{l}(r)=\psi_{l}\left(r_{1}\right)$, $\left(\frac{r}{p_{l}}\right)=\left(\frac{r_{1}}{p_{l}}\right), \exp \left(2 \pi i n r / p_{l}^{\beta+1}\right)=\exp \left(2 \pi i n r_{1} / p_{l}^{\beta+1}\right) \exp \left(2 \pi i n r_{2} / p_{l}^{\beta}\right)$ and

$$
\begin{aligned}
S_{2, k, l}\left(n, \psi_{l}\right)= & \sum_{\substack{r_{1} \bmod _{p_{l} \nmid r_{1}} \\
p_{l}}} \psi_{l}\left(r_{1}\right)\left(\frac{r_{1}}{p_{l}}\right)^{\beta+1} \\
& \times \exp \left(2 \pi i n r_{1} / p_{l}^{\beta+1}\right) \sum_{r_{2} \bmod p_{l}^{\beta}} \exp \left(2 \pi i n r_{2} / p_{l}^{\beta}\right) .
\end{aligned}
$$

Now the result follows from (3.10), (1.1) and (1.3).

Lemma 15. Let

$$
S_{3, k}(n, \chi)=\sum_{\substack{\omega=1 \\(2 N, \omega)=1}}^{\infty}\left(\chi(\omega)\left(\frac{-1}{\omega}\right) \varepsilon_{\omega}^{k} \omega^{-\frac{k}{2}} \sum_{r \bmod \omega}\left(\frac{r}{\omega}\right) \exp (2 \pi i n r / \omega)\right) .
$$

Then

$$
\begin{aligned}
S_{3, k}(n, \chi)= & n_{1}^{2-k} \frac{\mathcal{L}\left(\frac{k-1}{2}, \chi\left(\frac{(-1)^{\frac{k-1}{2}} u}{\cdot}\right)\right)}{\mathcal{L}\left(k-1, \chi^{2}\right)} \sum_{\delta d=n_{1}} \chi^{2}(\delta) d^{k-2} \\
& \times \prod_{q \mid d}\left(1-\left(\frac{(-1)^{\frac{k-1}{2}} u}{q}\right) \chi(q) q^{\frac{1-k}{2}}\right) .
\end{aligned}
$$


Proof. 1) First consider $S_{3, k}(u, \chi)$. Let $\omega=\omega_{0} \omega_{1}^{2}, \omega_{0}$ be square-free. Then by Lemma 7 we get

$$
\begin{aligned}
S_{3, k}(u, \chi)= & \sum_{\substack{\omega_{0}>0 \\
\left(2 N, \omega_{0}\right)=1 \\
q^{2} \nmid \omega_{0}}} \sum_{\substack{\omega_{1} \mid u \\
\left(2 N, \omega_{1}\right)=1}} \chi\left(\omega_{0}\right) \chi^{2}\left(\omega_{1}\right)\left(\frac{-1}{\omega_{0}}\right) \varepsilon_{\omega_{0}}^{k+1} \omega_{0}^{-\frac{k}{2}} \omega_{1}^{-k}\left(\frac{u}{\omega_{0}}\right) \sqrt{\omega_{0}} \omega_{1} \mu\left(\omega_{1}\right) \\
= & \sum_{\substack{\omega_{0}>0 \\
\left(2 N, \omega_{0}\right)=1 \\
q^{2} \nmid \omega_{0}}} \chi\left(\omega_{0}\right)\left(\frac{-u}{\omega_{0}}\right)\left(\frac{(-1)^{\frac{k+1}{2}}}{\omega_{0}}\right) \omega_{0}^{\frac{1-k}{2}} \sum_{\omega_{1} \mid u} \chi^{2}\left(\omega_{1}\right) \mu\left(\omega_{1}\right) \omega_{1}^{1-k} \\
= & \prod_{q \mid u}\left(1-\chi^{2}(q) q^{1-k}\right) \sum_{\substack{\omega_{0}>0 \\
q^{2} \nmid \omega_{0}}} \chi\left(\omega_{0}\right)\left(\frac{(-1)^{\frac{k-1}{2}} u}{\omega_{0}}\right) \omega_{0}^{\frac{1-k}{2}} .
\end{aligned}
$$

By (1.9) we have

$$
\begin{aligned}
& \mathcal{L}\left(\frac{k-1}{2}, \chi\left(\frac{(-1)^{\frac{k-1}{2}} u}{\cdot}\right)\right)=\sum_{\substack{\omega_{0}>0 \\
q^{2} \nmid \omega_{0}}} \sum_{\omega_{1}=1}^{\infty} \chi\left(\omega_{0}\right) \chi^{2}\left(\omega_{1}\right)\left(\frac{(-1)^{\frac{k-1}{2}} u}{\omega_{0} \omega_{1}^{2}}\right) \omega_{0}^{\frac{1-k}{2}} \omega_{1}^{1-k} \\
&= \sum_{\substack{\omega_{0}>0 \\
q^{2} \nmid \omega_{0}}} \chi\left(\omega_{0}\right)\left(\frac{(-1)^{\frac{k-1}{2}} u}{\omega_{0}}\right) \omega_{0}^{\frac{1-k}{2}} \sum_{\substack{\omega_{1}=1 \\
\left(u, \omega_{1}\right)=1}}^{\infty} \chi^{2}\left(\omega_{1}\right) \omega_{1}^{1-k} \\
&=\sum_{\substack{\omega_{0}>0 \\
q^{2} \nmid \omega_{0}}} \chi\left(\omega_{0}\right)\left(\frac{(-1)^{\frac{k-1}{2}} u}{\omega_{0}}\right) \omega_{0}^{\frac{1-k}{2}} \prod_{q \mid u}\left(1-\chi^{2}(q) q^{1-k}\right) \mathcal{L}\left(k-1, \chi^{2}\right) .
\end{aligned}
$$

(3.13) and (3.14) imply

$$
S_{3, k}(u, \chi)=\frac{\mathcal{L}\left(\frac{k-1}{2}, \chi\left(\frac{(-1)^{\frac{k-1}{2}} u}{\cdot}\right)\right)}{\mathcal{L}\left(k-1, \chi^{2}\right)} .
$$

2) Since $(2 N, \omega)=1$, from (3.11) we obtain

$$
S_{3, k}\left(2^{2 s} M u, \chi\right)=S_{3, k}(u, \chi) .
$$

3) Let $n=q^{2 \alpha} n_{0}, q^{2} \nmid n_{0}, q>2, q \neq p_{l}, l=1, \ldots, j, \omega=\omega_{0} q^{\beta}, q \nmid \omega_{0}$, $r=r_{1} q^{\beta}+r_{2} \omega_{0}, r_{1} \bmod \omega_{0}, r_{2} \bmod q^{\beta}$. It is easy to verify that if $2 \nmid a b$, $(a, b)=1$, then

$$
\varepsilon_{a b}=\left(\frac{a}{b}\right)\left(\frac{b}{a}\right) \varepsilon_{a} \varepsilon_{b} .
$$

Therefore $\varepsilon_{\omega_{0} q^{\beta}}^{k}=\left(\frac{\omega_{0}}{q}\right)^{\beta} \cdot\left(\frac{q}{\omega_{0}}\right)^{\beta} \varepsilon_{\omega_{0}}^{k} \varepsilon_{q^{\beta}}^{k}$.

By Lemma 4, from (3.11) we have

$$
S_{3, k}\left(n_{0}, \chi\right)=\sum_{\substack{\omega_{0}=1 \\\left(2 N q, \omega_{0}\right)=1}}^{\infty}\left(\chi\left(\omega_{0}\right)\left(\frac{-1}{\omega_{0}}\right) \varepsilon_{\omega_{0}}^{k} \omega_{0}^{-\frac{k}{2}}\right.
$$


ON THE FOURIER EXPANSIONS OF EISENSTEIN SERIES OF SOME TYPES

$$
\begin{aligned}
& \times \sum_{\beta=0}^{\infty} \chi^{\beta}(q)\left(\frac{-1}{q}\right)^{\beta} \cdot\left(\frac{q}{\omega_{0}}\right)^{\beta}\left(\frac{\omega_{0}}{q}\right)^{\beta} \varepsilon_{q^{\beta}}^{k} q^{-\frac{k}{2} \beta}\left(\frac{q}{\omega_{0}}\right)^{\beta}\left(\frac{\omega_{0}}{q}\right)^{\beta} \\
& \left.\times \sum_{r_{1} \bmod \omega_{0}}\left(\frac{r_{1}}{\omega_{0}}\right) \exp \left(2 \pi i n_{0} r_{1} / \omega_{0}\right) \sum_{r_{2} \bmod q^{\beta}}\left(\frac{r_{2}}{q}\right)^{\beta} \exp \left(2 \pi i n_{0} r_{2} / q^{\beta}\right)\right) \\
= & \sum_{\substack{\omega_{0}=1 \\
\left(2 N q \omega_{0}\right)=1}}^{\infty}\left(\chi\left(\omega_{0}\right)\left(\frac{-1}{\omega_{0}}\right) \varepsilon_{\omega_{0}}^{k} \omega_{0}^{-\frac{k}{2}} \sum_{r_{1} \bmod \omega_{0}}\left(\frac{r_{1}}{\omega_{0}}\right) \exp \left(2 \pi i n_{0} r_{1} / \omega_{0}\right)\right) \\
& \times \sum_{\beta=0}^{\infty}\left(\chi^{\beta}(q)\left(\frac{-1}{q}\right)^{\beta} q^{-\frac{k}{2} \beta} \varepsilon_{q^{\beta}}^{k} \sum_{r_{2} \bmod q^{\beta}}\left(\frac{r_{2}}{q}\right)^{\beta} \exp \left(2 \pi i n_{0} r_{2} / q^{\beta}\right)\right) \\
= & A_{1}\left(n_{0}\right) A_{2}\left(n_{0}\right) .
\end{aligned}
$$

Let $r_{2}=r_{3}+q r_{4}, r_{3} \bmod q, r_{4} \bmod q^{\beta-1}$. Then

$$
\begin{aligned}
A_{2}\left(n_{0}\right)= & 1+\sum_{\beta=1}^{\infty}\left(\chi^{\beta}(q)\left(\frac{-1}{q}\right)^{\beta} q^{-\frac{k}{2} \beta} \varepsilon_{q^{\beta}}^{k} \sum_{r_{3} \bmod q}\left(\frac{r_{3}}{q}\right)^{\beta} \exp \left(2 \pi i n_{0} r_{3} / q^{\beta}\right)\right. \\
& \left.\times \sum_{r_{4} \bmod q^{\beta-1}} \exp \left(2 \pi i n_{0} r_{4} / q^{\beta-1}\right)\right) .
\end{aligned}
$$

Since $q^{2} \nmid n_{0}$, by (1.3), (1.1) and Lemma 3 we get

$$
\begin{aligned}
A_{2}\left(n_{0}\right)= & 1+\chi(q)\left(\frac{-1}{q}\right) q^{-\frac{k}{2}} \varepsilon_{q}^{k} \sum_{r_{3} \bmod q}\left(\frac{r_{3}}{q}\right) \exp \left(2 \pi i n_{0} r_{3} / q\right) \\
& +\chi^{2}(q) q^{-k} \sum_{r_{3} \bmod q} \exp \left(2 \pi i n_{0} r_{3} / q^{2}\right) \sum_{r_{4} \bmod q} \exp \left(2 \pi i n_{0} r_{4} / q\right) \\
= & \begin{cases}1+\chi(q)\left(\frac{(-1)^{\frac{k-1}{2}} n_{0}}{q}\right) q^{\frac{1-k}{2}} & \text { if } q \nmid n_{0} ; \\
1-\chi^{2}(q) q^{1-k} & \text { if } q \mid n_{0} .\end{cases}
\end{aligned}
$$

Similarly to $(3.18)$

$$
S_{3, k}\left(q^{2 \alpha} n_{0}, \chi\right)=A_{1}\left(q^{2 \alpha} n_{0}\right) A_{2}\left(q^{2 \alpha} n_{0}\right)=A_{1}\left(n_{0}\right) A_{2}\left(q^{2 \alpha} n_{0}\right),
$$

because $q \nmid \omega_{0}$.

As in the case of (3.19),

$$
\begin{aligned}
A_{2}\left(q^{2 \alpha} n_{0}\right)= & 1+\sum_{\beta=1}^{\infty}\left(\chi^{\beta}(q)\left(\frac{-1}{q}\right)^{\beta} q^{-\frac{k}{2} \beta} \varepsilon_{q^{\beta}}^{k} \sum_{r_{3} \bmod q}\left(\frac{r_{3}}{q}\right)^{\beta} \exp \left(2 \pi i q^{2 \alpha} n_{0} r_{3} / q^{\beta}\right)\right. \\
& \left.\times \sum_{r_{4} \bmod q^{\beta-1}} \exp \left(2 \pi i q^{2 \alpha} n_{0} r_{4} / q^{\beta-1}\right)\right) .
\end{aligned}
$$

By (1.3), (1.1), Lemma 3 and (3.20), from (3.22) we obtain: 
a) if $q \mid n_{0}$, then

$$
\begin{aligned}
& A_{2}\left(q^{2 \alpha} n_{0}\right)=1+\sum_{\beta=1}^{\alpha} \chi^{2 \beta}(q) q^{(2-k) \beta-1}(q-1)-\chi^{2 \alpha+2}(q) q^{(2-k) \alpha} q^{1-k} \\
& =\left(1-\chi^{2}(q) q^{1-k}\right) \chi^{2 \alpha}(q) q^{(2-k) \alpha}-\chi^{2 \alpha}(q) q^{(2-k) \alpha} \\
& \quad+\sum_{\beta=0}^{\alpha} \chi^{2 \beta}(q) q^{(2-k) \beta}-\chi^{2}(q) q^{1-k} \sum_{\beta=1}^{\alpha} \chi^{2(\beta-1)}(q) q^{(2-k)(\beta-1)} \\
& =\left(1-\chi^{2}(q) q^{1-k}\right) \sum_{\beta=0}^{\alpha} \chi^{2 \beta}(q) q^{(2-k) \beta}=A_{2}\left(n_{0}\right) q^{(2-k) \alpha} \sum_{\beta=0}^{\alpha} \chi^{2 \beta}(q) q^{(k-2)(\alpha-\beta)} \\
& =A_{2}\left(n_{0}\right) q^{(2-k) \alpha} \sum_{\delta d=q^{\alpha}} \chi^{2}(\delta) d^{k-2}
\end{aligned}
$$

b) if $q \nmid n_{0}$, then

$$
\begin{aligned}
& A_{2}\left(q^{2 \alpha} n_{0}\right)=1+\sum_{\beta=1}^{\alpha} \chi^{2 \beta}(q) q^{(2-k) \beta-1}(q-1)+\chi^{2 \alpha+1}(q) q^{(2-k) \alpha} q^{\frac{1-k}{2}}\left(\frac{(-1)^{\frac{k-1}{2}} n_{0}}{q}\right) \\
&=\left(1+\chi(q)\left(\frac{(-1)^{\frac{k-1}{2}} n_{0}}{q}\right) q^{\frac{1-k}{2}}\right) \chi^{2 \alpha}(q) q^{(2-k) \alpha}-\chi^{2 \alpha}(q) q^{(2-k) \alpha} \\
&+\sum_{\beta=0}^{\alpha} \chi^{2 \beta}(q) q^{(2-k) \beta}-\chi^{2}(q) q^{1-k} \sum_{\beta=1}^{\alpha} \chi^{2(\beta-1)}(q) q^{(2-k)(\beta-1)} \\
&=\left(1+\chi(q)\left(\frac{(-1)^{\frac{k-1}{2}} n_{0}}{q}\right) q^{\frac{1-k}{2}}\right) \chi^{2 \alpha}(q) q^{(2-k) \alpha} \\
&+\left(1-\chi^{2}(q) q^{1-k}\right) \sum_{\beta=0}^{\alpha-1} \chi^{2 \beta}(q) q^{(2-k) \beta} \\
&=\left(1+\chi(q)\left(\frac{(-1)^{\frac{k-1}{2}} n_{0}}{q}\right) q^{\frac{1-k}{2}}\right)\left(\chi^{2 \alpha}(q) q^{(2-k) \alpha}\right. \\
&\left.+\left(1-\chi(q)\left(\frac{(-1)^{\frac{k-1}{2}} n_{0}}{q}\right) q^{\frac{1-k}{2}}\right) q^{(2-k) \alpha} \sum_{\beta=0}^{\alpha-1} \chi^{2 \beta}(q) q^{(k-2)(\alpha-\beta)}\right) \\
&= A_{2}\left(n_{0}\right) q^{(2-k) \alpha} \sum_{\delta d=q^{\alpha}} \chi^{2}(\delta) d^{k-2} \prod_{q_{1} \mid d}\left(1-\chi\left(q_{1}\right)\left(\frac{(-1)^{\frac{k-1}{2}} n_{0}}{q_{1}}\right) q_{1}^{\frac{1-k}{2}}\right) . \quad(3.24)
\end{aligned}
$$

If $q_{1} \mid n_{0}$, then $\left(\frac{(-1)^{\frac{k-1}{2}} n_{0}}{q_{1}}\right)=0$, therefore (3.24) contains (3.23).

Taking into account that $f_{1}(\delta)=\chi^{2}(\delta), f_{2}(\delta)=\delta^{k-2}$ and $f_{3}(d)=\prod_{q_{1} \mid d}(1-$ $\left.\chi\left(q_{1}\right)\left(\frac{(-1)^{\frac{k-1}{2}} n_{0}}{q_{1}}\right) q_{1}^{\frac{1-k}{2}}\right)$ are multiplicative functions, from (3.15), (3.16), (3.21) and (3.24) follows (3.12). 
Note that if $\chi=\chi_{0}$, then by (1.9), (1.10)

$$
\begin{gathered}
\mathcal{L}\left(\frac{k-1}{2}, \chi_{0}\left(\frac{(-1)^{\frac{k-1}{2}} u}{\cdot}\right)\right) \\
=\prod_{l=1}^{j}\left(1-\left(\frac{(-1)^{\frac{k-1}{2}} u}{p_{l}}\right) p_{l}^{\frac{1-k}{2}}\right) \mathcal{L}\left(\frac{k-1}{2},(-1)^{\frac{k-1}{2}} u\right) ; \\
\mathcal{L}\left(k-1, \chi_{0}^{2}\right)=\prod_{l=1}^{j}\left(1-p_{l}^{1-k}\right)\left(1-2^{1-k}\right) \zeta(k-1) ;
\end{gathered}
$$

if $\chi=\left(\frac{4 N}{\cdot}\right)$, then $\chi^{2}=\chi_{0}$ and by $(1.9),(1.10)$

$$
\mathcal{L}\left(\frac{k-1}{2}, \chi\left(\frac{(-1)^{\frac{k-1}{2}} u}{\cdot}\right)\right)=\mathcal{L}\left(\frac{k-1}{2},(-1)^{\frac{k-1}{2}} u N\right) .
$$

Proposition 2. Let $\chi(n)=\phi(n) \psi_{1, l}(n) \psi_{2, l}(n)$, where $\psi_{1, l}$ is a character $\bmod N_{l}\left(N_{l} \mid N\right)$ and $\psi_{2, l}$ is a character $\bmod N / N_{l}, \chi(-1)=1,2 \nmid k$; then the system of functions

$$
\begin{gathered}
E_{1}\left(\tau ; \frac{k}{2}, 4 N, \chi\right)=\sum_{\substack{4 N \mid m \\
n>0,(m, n)=1}} \bar{\chi}(n)\left(\frac{m}{n}\right) \varepsilon_{n}^{k}(m \tau+n)^{-k / 2}, \\
E_{2, l}\left(\tau ; \frac{k}{2}, 4 N, \chi\right)=\sum_{\substack{m>0, n \\
\left(2 N_{l} m, N n / N_{l}\right)=1}} \phi(n) \bar{\psi}_{1, l}(n) \psi_{2, l}(m)\left(\frac{4 N_{l} m}{n}\right) \\
\times \varepsilon_{n}^{k}\left(4 N_{l} m \tau+n\right)^{-k / 2}
\end{gathered}
$$

$\left(N_{l} \mid N, \quad N_{l} \neq N, \quad l=1, \ldots, 2^{j}-1, \quad N_{l_{1}} \neq N_{l_{2}}\right.$, when $\left.l_{1} \neq l_{2}\right)$,

$$
\begin{aligned}
E_{3, l}\left(\tau ; \frac{k}{2}, 4 N, \chi\right)=\sum_{\substack{m>0, n \\
\left(N_{l} m, 2 N n / N_{l}\right)=1}} \phi(m) \bar{\psi}_{1, l}(n) \psi_{2, l}(m)\left(\frac{-n}{m N_{l}}\right) \\
\times \varepsilon_{m N_{l}}^{k}\left(N_{l} m \tau+n\right)^{-k / 2}
\end{aligned}
$$

$$
\left(N_{l} \mid N, \quad l=1, \ldots, 2^{j}, \quad N_{l_{1}} \neq N_{l_{2}}, \text { when } l_{1} \neq l_{2}\right)
$$

is the basis of the space $E_{k / 2}\left(\widetilde{\Gamma}_{0}(4 N), \chi\right)$ for any odd $k \geq 5$.

This proposition is proved exactly as Proposition 1.

Next, we derive Fourier expansions of functions (3.28)-(3.30).

Let $a_{k}=\pi^{k / 2} \exp (-\pi i k / 4) \Gamma\left(\frac{k}{2}\right)^{-1}$.

1) if $2 \nmid n, n<0$, then by (3.17) we obtain

$$
\varepsilon_{-n}=\left(\frac{-1}{n}\right)\left(\frac{n}{-1}\right) \varepsilon_{n} \varepsilon_{-1}=-\left(\frac{-1}{|n|}\right)(-1) \varepsilon_{n} \cdot i=\left(\frac{-1}{-n}\right) i \varepsilon_{n} .
$$

It is easy to verify that if $m>0, n<0, \tau \in \mathbb{H}$, then

$$
\sqrt{-m \tau-n} \cdot i=\sqrt{m \tau+n} \text {. }
$$


Since $\bar{\chi}(-n)=\bar{\chi}(n)$, by (3.31) and (3.32) we have

$$
\sum_{\substack{4 N \mid m, n>0 \\ m<0,(m, n)=1}} \bar{\chi}(n)\left(\frac{m}{n}\right) \varepsilon_{n}^{k}(m \tau+n)^{-k / 2}=\sum_{\substack{4 N \mid m, n<0 \\ m>0,(m, n)=1}} \bar{\chi}(n)\left(\frac{m}{n}\right) \varepsilon_{n}^{k}(m \tau+n)^{-k / 2}
$$

therefore

$$
\begin{aligned}
E_{1}\left(\tau ; \frac{k}{2}, 4 N, \chi\right) & =1+\sum_{\substack{4 N \mid m, m>0 \\
n,(m, n)=1}} \bar{\chi}(n)\left(\frac{m}{n}\right) \varepsilon_{n}^{k}(m \tau+n)^{-k / 2} \\
& =1+\sum_{\substack{m>0 \\
n,(2 m N, n)=1}} \bar{\chi}(n)\left(\frac{4 N m}{n}\right) \varepsilon_{n}^{k}(4 N m \tau+n)^{-k / 2} .
\end{aligned}
$$

Let $n=r+4 N m h, r \bmod 4 N m, h \in \mathbb{Z}$. Then $\bar{\chi}(n)=\bar{\chi}(r), \varepsilon_{n}=\varepsilon_{r}$ and by Lemma 5 we get

$$
\begin{aligned}
& E_{1}\left(\tau ; \frac{k}{2}, 4 N, \chi\right)=1+(4 N)^{-\frac{k}{2}} \sum_{m=1}^{\infty}\left(m^{-\frac{k}{2}} \sum_{\substack{r \bmod 4 N m \\
(2 N, r)=1}} \bar{\chi}(r)\left(\frac{N}{r}\right)\left(\frac{m}{r}\right) \varepsilon_{r}^{k}\right. \\
& \left.\times \sum_{h=-\infty}^{\infty}\left(\tau+\frac{r}{4 N m}+h\right)^{-\frac{k}{2}}\right) \\
& =1+(2 N)^{-\frac{k}{2}} \pi^{\frac{k}{2}} \exp \left(-\frac{\pi i k}{4}\right) \Gamma\left(\frac{k}{2}\right)^{-1} \sum_{n=1}^{\infty}\left(n^{\frac{k}{2}-1} \sum_{m=1}^{\infty} m^{-\frac{k}{2}}\right. \\
& \left.\times \sum_{\substack{r \bmod 4 N m \\
(2 N, r)=1}} \bar{\chi}(r)\left(\frac{N}{r}\right)\left(\frac{m}{r}\right) \varepsilon_{r}^{k} \exp (2 \pi i n r /(4 N m))\right) z^{n} \\
& =1+(2 N)^{-\frac{k}{2}} a_{k} \sum_{n=1}^{\infty}\left(n^{\frac{k}{2}-1} \sum_{\alpha=0}^{\infty} \sum_{\beta_{1}=0}^{\infty} \cdots \sum_{\beta_{j}=0}^{\infty} \sum_{\substack{\omega=1 \\
(2 N, \omega)=1}}^{\infty}\left(2^{\alpha} p_{1}^{\beta_{1}} \cdots p_{j}^{\beta_{j}} \omega\right)^{-\frac{k}{2}}\right. \\
& \times \sum_{\substack{r \bmod 2^{\alpha+2} p_{1}^{\beta_{1}+1} \ldots p_{j}^{\beta_{j}+1} \omega \\
(2 N, r)=1}} \bar{\chi}(r)\left(\frac{2}{r}\right)^{\alpha}\left(\frac{p_{1}}{r}\right)^{\beta_{1}+1} \cdots\left(\frac{p_{j}}{r}\right)^{\beta_{j}+1}\left(\frac{\omega}{r}\right) \varepsilon_{r}^{k} \\
& \left.\times \exp \left(2 \pi i n r /\left(2^{\alpha+2} p_{1}^{\beta_{1}+1} \cdots p_{j}^{\beta_{j}+1} \omega\right)\right)\right) z^{n} .
\end{aligned}
$$

Then, as in Proposition 1,

$$
\begin{gathered}
r=\omega \cdot 2^{\alpha+2} \sum_{h=1}^{j} r_{h} \prod_{\substack{h_{1}=1 \\
h_{1} \neq h}}^{j} p_{h_{1}}^{\beta_{h_{1}}+1}+\omega r_{j+1} \prod_{h=1}^{j} p_{h}^{\beta_{h}+1}+2^{\alpha+2} r_{j+2} \prod_{h=1}^{j} p_{h}^{\beta_{h}+1}, \\
0 \leq r_{j+2}<\omega, \quad 0 \leq r_{h}<p_{h}^{\beta_{h}+1}, \quad 1 \leq h \leq j, \quad 0 \leq r_{j+1}<2^{\alpha+2} .
\end{gathered}
$$

$2 \nmid r_{j+1}, p_{h} \nmid r_{h}$, since $(2 N, r)=1$. After a simple calculation we obtain 
ON THE FOURIER EXPANSIONS OF EISENSTEIN SERIES OF SOME TYPES

$$
\begin{gathered}
\bar{\chi}(r)\left(\frac{2}{r}\right)^{\alpha}\left(\frac{p_{1}}{r}\right)^{\beta_{1}+1} \ldots\left(\frac{p_{j}}{r}\right)^{\beta_{j}+1}\left(\frac{\omega}{r}\right) \varepsilon_{r}^{k} \\
\quad \times \exp \left(2 \pi i n r /\left(2^{\alpha+2} p_{1}^{\beta_{1}+1} \ldots p_{j}^{\beta_{j}+1} \omega\right)\right)=\phi(N) \bar{\psi}(4) \bar{\chi}(\omega) \\
\times\left(\frac{-1}{\omega}\right) \varepsilon_{\omega}^{k}\left(\frac{r_{j+2}}{\omega}\right) \exp \left(2 \pi i n r_{j+2} / \omega\right) \bar{\psi}^{\alpha}(2) \phi\left(r_{j+1}\right)\left(\frac{2}{r_{j+1}}\right)^{\alpha} \varepsilon_{r_{j+1}}^{k} \\
\times \exp \left(2 \pi i n r_{j+1} / 2^{\alpha+2}\right) \prod_{h=1}^{j}\left(\phi^{\beta_{h}}\left(p_{h}\right)\left(\frac{-1}{p_{h}}\right)^{\beta_{h}+1} \varepsilon_{p_{h}^{\beta_{h}+1}} \bar{\psi}_{h}\left(r_{h}\right)\left(\frac{r_{h}}{p_{h}}\right)^{\beta_{h}+1}\right. \\
\times \prod_{\substack{h_{1}=1 \\
h_{1} \neq h}}^{\left.\left(\frac{p_{h_{1}}}{p_{h}}\right)^{\left(\beta_{h_{1}}+1\right)\left(\beta_{h}+1\right)} \bar{\psi}_{h}\left(p_{h_{1}}^{\beta_{h_{1}}+1}\right) \exp \left(2 \pi i n r_{h} / p_{h}^{\beta_{h}+1}\right)\right)}
\end{gathered}
$$

From (3.33) and (3.34) we have

$$
\begin{aligned}
& E_{1}\left(\tau ; \frac{k}{2}, 4 N, \chi\right)=1+(2 N)^{-\frac{k}{2}} a_{k} \sum_{n=1}^{\infty}\left(n^{\frac{k}{2}-1} \phi(N) \bar{\psi}(4)\right. \\
& \times \sum_{\substack{\omega=1 \\
(2 N, \omega)=1}}^{\infty} \omega^{-\frac{k}{2}} \bar{\chi}(\omega)\left(\frac{-1}{\omega}\right) \varepsilon_{\omega}^{k} \sum_{r_{j+2} \bmod \omega}\left(\frac{r_{j+2}}{\omega}\right) \exp \left(2 \pi i n r_{j+2} / \omega\right) \\
& \times \sum_{\alpha=0}^{\infty} 2^{-\frac{k}{2} \alpha \bar{\psi}^{\alpha}}(2) \sum_{\substack{r_{j+1} \bmod _{2 \nmid r_{j+1}}{ }^{\alpha+2} \\
r_{j+1}}} \phi\left(r_{j+1}\right)\left(\frac{2}{r_{j+1}} \varepsilon_{r_{j+1}}^{k} \exp \left(2 \pi i n r_{j+1} / 2^{\alpha+2}\right)\right. \\
& \times \sum_{\beta_{1}=0}^{\infty} \cdots \sum_{\beta_{j}=0}^{\infty} \prod_{h=1}^{j}\left(p_{h}^{-\frac{k}{2} \beta_{h}} \phi^{\beta_{h}}\left(p_{h}\right)\left(\frac{-1}{p_{h}}\right)^{\beta_{h}+1} \varepsilon_{p_{h}^{\beta_{h}+1}}^{k}\right. \\
& \times \prod_{\substack{h_{1}=1 \\
h_{1} \neq h}}^{j}\left(\frac{p_{h_{1}}}{p_{h}}\right)^{\left(\beta_{h_{1}}+1\right)\left(\beta_{h}+1\right)} \bar{\psi}_{h_{1}}\left(p_{h}^{\beta_{h}+1}\right) \\
& \left.\left.\times \sum_{\substack{r_{h} \bmod p_{h}^{\beta_{h}+1} \\
p_{h} \nmid r_{h}}} \bar{\psi}_{h}\left(r_{h}\right)\left(\frac{r_{h}}{p_{h}}\right)^{\beta_{h}+1} \exp \left(2 \pi i n r_{h} / p_{h}^{\beta_{h}+1}\right)\right)\right) z^{n} \\
& =1+(2 N)^{-k / 2} a_{k} \phi(N) \bar{\psi}(4) \sum_{n=1}^{\infty}\left(n^{\frac{k}{2}-1} S_{1, k}(n, \bar{\chi}) S_{3, k}(n, \bar{\chi})\right. \\
& \times \sum_{\beta_{1}=0}^{2 t_{1}+1} \cdots \sum_{\beta_{j}=0}^{2 t_{j}+1} \prod_{h=1}^{j}\left(p_{h}^{-\frac{k}{2} \beta_{h}} \phi^{\beta_{h}}\left(p_{h}\right)\left(\frac{-1}{p_{h}}\right)^{\beta_{h}+1} \varepsilon_{p_{h}^{\beta_{h}+1}}^{k}\right. \\
& \left.\left.\times \prod_{\substack{h_{1}=1 \\
h_{1} \neq h}}^{j}\left(\frac{p_{h_{1}}}{p_{h}}\right)^{\left(\beta_{h_{1}}+1\right)\left(\beta_{h}+1\right)} \bar{\psi}_{h_{1}}\left(p_{h}^{\beta_{h}+1}\right) S_{2, k, h}\left(n, \bar{\psi}_{h}\right)\right)\right) z^{n} .
\end{aligned}
$$


2) Let $\chi_{1}=\phi \bar{\psi}_{1, l} \psi_{2, l}$; if $N_{l}=1, d=0$ or $N_{l}=p_{\delta_{1}} \cdots p_{\delta_{d}}, 1 \leq d \leq j-1$, then similarly to the above we obtain

$$
\begin{aligned}
& E_{2, l}\left(\tau ; \frac{k}{2}, 4 N, \chi\right)=\left(2 N_{l}\right)^{-k / 2} a_{k} \phi\left(N_{l}\right) \bar{\psi}_{1, l}(4) \sum_{n=1}^{\infty}\left(n^{\frac{k}{2}-1} S_{1, k}\left(n, \chi_{1}\right) S_{3, k}\left(n, \chi_{1}\right)\right. \\
& \times \sum_{\beta_{1}=0}^{2 t_{\delta_{1}}+1} \cdots \sum_{\beta_{d}=0}^{2 t_{\delta_{d}}+1} \prod_{h=1}^{d}\left(p_{\delta_{h}}^{-\frac{k}{2} \beta_{h}} \phi^{\beta_{h}}\left(p_{\delta_{h}}\right)\left(\frac{-1}{p_{\delta_{h}}}\right)^{\beta_{h}+1} \varepsilon_{p_{\delta_{h}}^{\beta_{h}+1}}^{k}\right. \\
&\left.\left.\quad \times \prod_{\substack{h_{1}=1 \\
h_{1} \neq h}}^{d}\left(\frac{p_{\delta_{h_{1}}}}{p_{\delta_{h}}}\right)^{\left(\beta_{h_{1}}+1\right)\left(\beta_{h}+1\right)} \bar{\psi}_{\delta_{h}}\left(p_{\delta_{h_{1}}}^{\beta_{h_{1}}+1}\right) S_{2, k, \delta_{h}}\left(n, \bar{\psi}_{\delta_{h}}\right)\right)\right) z^{n}
\end{aligned}
$$

and if $N_{l}=1, d=0$ or $N_{l}=p_{\delta_{1}} \cdots p_{\delta_{d}}, 1 \leq d \leq j$, then

$$
\begin{aligned}
E_{3, l} & \left(\tau ; \frac{k}{2}, 4 N, \chi\right)=2^{k / 2} N_{l}^{-k / 2} a_{k} \sum_{n=1}^{\infty}\left(n^{\frac{k}{2}-1} S_{3, k}\left(n, \chi_{1}\right)\right. \\
& \left.\times \sum_{\beta_{1}=0}^{2 t_{\delta_{1}}+1} \cdots \sum_{\beta_{d}=0}^{2 t_{\delta_{d}}+1} \prod_{h=1}^{d}\left(p_{\delta_{h}}^{-\frac{k}{2} \beta_{h}} \phi^{\beta_{h}}\left(p_{\delta_{h}}\right)\left(\frac{-1}{p_{\delta_{h}}}\right)^{\beta_{h}+1} \varepsilon_{p_{\delta_{h}}^{\beta_{h}+1}}^{k} \bar{\psi}_{\delta_{h}}\left(p_{\delta_{h_{1}}}^{\beta_{h_{1}}+1}\right) S_{2, k, \delta_{h}}\left(n, \bar{\psi}_{\delta_{h}}\right)\right)\right) z^{n} . \\
& \times \prod_{\substack{h_{1}=1 \\
h_{1} \neq h}}^{d}\left(\frac{p_{\delta_{h}}}{p_{\delta_{h_{1}}}}\right)^{\left(\beta_{h_{1}}+1\right)\left(\beta_{h}+1\right)}
\end{aligned}
$$

For $N=p$, we obtain the following results.

Let now $n=2^{2 s} p^{2 t} n_{1}^{2} u,\left(2 p, n_{1}\right)=1, q^{2} \nmid u$,

$$
\begin{aligned}
& A_{1, k}(u, \chi)=\frac{\pi^{\frac{k}{2}} u^{\frac{k}{2}-1}}{\Gamma\left(\frac{k}{2}\right) 2^{\frac{k-1}{2}}}\left(\frac{2}{k}\right) \frac{\mathcal{L}\left(\frac{k-1}{2}, \chi\left(\frac{(-1)^{\frac{k-1}{2}} u}{\cdot}\right)\right)}{\mathcal{L}\left(k-1, \chi^{2}\right)} \sum_{\delta d=n_{1}} \chi^{2}(\delta) d^{k-2} \\
& \times \prod_{q \mid d}\left(1-\left(\frac{(-1)^{\frac{k-1}{2}} u}{q}\right) \chi(q) q^{\frac{1-k}{2}}\right), \\
& d_{1, k}(u)= \begin{cases}-1 & \text { when } p \mid u, \\
\left(\frac{(-1)^{\frac{k-1}{2}} u}{p}\right) p^{\frac{k-1}{2}} & \text { when } p \nmid u,\end{cases} \\
& d_{2, k}(u)= \begin{cases}-1 & \text { when } p \nmid u, \\
p-1+\left(\frac{(-1)^{\frac{k-1}{2}} u / p}{p}\right) p^{\frac{3-k}{2}} & \text { when } p \mid u,\end{cases} \\
& d_{3, k}(u)= \begin{cases}\left(\frac{(-1)^{\frac{k+1}{2}} u}{p}\right) \psi(u) \varepsilon_{p} \sqrt{p} g\left(\bar{\psi}\left(\frac{\dot{p}}{p}\right)\right) & \text { when } p \nmid u, \\
\phi(p) \psi\left(\frac{u}{p}\right) p^{\frac{3-k}{2}} g(\bar{\psi}) & \text { when } p \mid u,\end{cases}
\end{aligned}
$$




$$
\begin{aligned}
A_{k}(u)= & \frac{2^{\frac{k+1}{2}}\left(\frac{k-1}{2}\right) ! u^{\frac{k}{2}-1}}{\left(p^{k-1}-1\right)\left(2^{k-1}-1\right) \pi^{\frac{k-1}{2}} B_{\frac{k-1}{2}}}\left(\frac{2}{k}\right)\left(1-\left(\frac{(-1)^{\frac{k-1}{2}} u}{p}\right) p^{\frac{1-k}{2}}\right) \\
& \times \mathcal{L}\left(\frac{k-1}{2},(-1)^{\frac{k-1}{2}} u\right) \sum_{d \mid n_{1}} d^{k-2} \prod_{q \mid d}\left(1-\left(\frac{(-1)^{\frac{k-1}{2}} u}{q}\right) q^{\frac{1-k}{2}}\right),
\end{aligned}
$$

where $B_{\frac{k-1}{2}}$ are Bernoulli numbers;

$$
\begin{gathered}
B_{r, k}(u)=p^{k-2}(p-1) \cdot \frac{p^{(k-2) t}-1}{p^{k-2}-1}+d_{r, k}(u) \quad(r=1,2), \\
C_{1, k}(u, \chi)=2^{k-2} \bar{\psi}(4) \cdot \frac{2^{(k-2) s} \bar{\psi}^{s}(4)-1}{2^{k-2} \bar{\psi}(4)-1}+c_{k}(u, \chi),
\end{gathered}
$$

where $c_{k}(u, \chi)$ is defined by Lemma 13 .

Corollary 5. If $\chi \neq \bar{\chi}, \chi(-1)=1$, then

$$
\begin{aligned}
E_{1}\left(\tau ; \frac{k}{2}, 4 p, \chi\right)= & 1+\frac{\left(1+\phi(3)\left(\frac{-1}{k}\right) i\right) p^{-\frac{k}{2}}}{\left(1+\left(\frac{-1}{k}\right) i\right) \psi(4)} \phi(p) \\
& \times \sum_{n=1}^{\infty} A_{1, k}(u, \bar{\chi}) C_{1, k}(u, \bar{\chi}) \psi^{2}\left(n_{1}\right) d_{3, k}(u) z^{n}, \\
E_{2}\left(\tau ; \frac{k}{2}, 4 p, \chi\right)= & \frac{1+\phi(3)\left(\frac{-1}{k}\right) i}{1+\left(\frac{-1}{k}\right) i} \sum_{n=1}^{\infty} p^{(k-2) t} \psi^{s}(4) A_{1, k}(u, \chi) C_{1, k}(u, \chi) z^{n}, \\
E_{3,1}\left(\tau ; \frac{k}{2}, 4 p, \chi\right)= & \frac{2^{k}}{1+\left(\frac{-1}{k}\right) i} \sum_{n=1}^{\infty} 2^{(k-2) s} p^{(k-2) t} A_{1, k}(u, \chi) z^{n}, \\
E_{3,2}\left(\tau ; \frac{k}{2}, 4 p, \chi\right)= & \frac{2^{k} p^{-k / 2}}{1+\left(\frac{-1}{k}\right) i} \sum_{n=1}^{\infty} 2^{(k-2) s} \psi^{s}(4) \psi^{2}\left(n_{1}\right) A_{1, k}(u, \bar{\chi}) d_{3, k}(u) z^{n} .
\end{aligned}
$$

Proof immediately follows from (3.35)-(3.37) and Lemmas 13-15, since $\exp \left(\frac{\pi i k}{4}\right)=\left(1+\left(\frac{-1}{k}\right) i\right)\left(\frac{2}{k}\right) \cdot 2^{-\frac{1}{2}}$ when $2 \nmid k$.

Corollary 6. If $\chi=\chi_{0}$, then

$$
\begin{aligned}
E_{1}\left(\tau ; \frac{k}{2}, 4 p, \chi_{0}\right) & =1+\sum_{n=1}^{\infty} A_{k}(u) B_{1, k}(u) C_{1, k}\left(u, \chi_{0}\right) z^{n} \\
E_{2}\left(\tau ; \frac{k}{2}, 4 p, \chi_{0}\right) & =p^{k-1} \sum_{n=1}^{\infty} p^{(k-2) t} A_{k}(u) C_{1, k}\left(u, \chi_{0}\right) z^{n} \\
E_{3,1}\left(\tau ; \frac{k}{2}, 4 p, \chi_{0}\right) & =2^{k} p^{k-1}\left(1+\left(\frac{-1}{k}\right) i\right)^{-1} \sum_{n=1}^{\infty} 2^{(k-2) s} p^{(k-2) t} A_{k}(u) z^{n} \\
E_{3,2}\left(\tau ; \frac{k}{2}, 4 p, \chi_{0}\right) & =2^{k}\left(1+\left(\frac{-1}{k}\right) i\right)^{-1} \sum_{n=1}^{\infty} 2^{(k-2) s} A_{k}(u) B_{1, k}(u) z^{n} .
\end{aligned}
$$


Proof directly follows from (3.35)-(3.37) and Lemmas 13-15, taking $\chi=\chi_{0}$.

Corollary 7. If $\chi(n)=\left(\frac{4 p}{n}\right)$, then

$$
\begin{aligned}
E_{1}\left(\tau ; \frac{k}{2}, 4 p, \chi\right) & =1+\sum_{n=1}^{\infty} A_{k}(u p) B_{2, k}(u) C_{1, k}(u, \chi) z^{n} \\
E_{2}\left(\tau ; \frac{k}{2}, 4 p, \chi\right) & =p^{k / 2} \frac{1+\left(\frac{-1}{p k}\right) i}{1+\left(\frac{-1}{k}\right) i} \sum_{n=1}^{\infty} p^{(k-2) t} A_{k}(u p) C_{1, k}(u, \chi) z^{n} \\
E_{3,1}\left(\tau ; \frac{k}{2}, 4 p, \chi\right) & =2^{k} p^{k / 2}\left(1+\left(\frac{-1}{k}\right) i\right)^{-1} \sum_{n=1}^{\infty} 2^{(k-2) s} p^{(k-2) t} A_{k}(u p) z^{n}, \\
E_{3,2}\left(\tau ; \frac{k}{2}, 4 p, \chi\right) & =2^{k} \varepsilon_{p}\left(\frac{-1}{p}\right)^{\frac{k+1}{2}}\left(1+\left(\frac{-1}{k}\right) i\right)^{-1} \sum_{n=1}^{\infty} 2^{(k-2) s} A_{k}(u p) B_{2, k}(u) z^{n} .
\end{aligned}
$$

Proof. If $p \equiv 1(\bmod 4)$, then $\chi(d)=\phi_{0}(d)\left(\frac{d}{p}\right)$, and if $p \equiv-1(\bmod 4)$, then $\chi(d)=\left(\frac{-1}{d}\right)\left(\frac{d}{p}\right)$. Therefore $\chi(d)=\left(\frac{-1}{d}\right)^{\frac{p-1}{2}} \cdot\left(\frac{d}{p}\right),\left(\frac{-1}{p}\right)^{\frac{p-1}{2}}=\left(\frac{-1}{p}\right)$ and the result follows from (3.35)-(3.37) and Lemmas 13-15.

\section{REFERENCES}

1. N. Koblitz, Introduction to elliptic curves and modular forms. Graduate Texts in Mathematics, 97. Springer-Verlag, New York, 1984.

2. A. G. VAn Asch, Modular forms of half integral weight, some explicit arithmetic. Math. Ann. 262(1983), No. 1, 77-89.

3. T. Y. PeI, Eisenstein series of weight 3/2. I. Trans. Amer. Math. Soc. 274(1982), No. 2, 573-606.

4. N. G. Chudakov, Introduction to the theory of Dirichlet's $L$-functions. (Russian) OGIZ, Moscow-Leningrad, 1947.

5. B. SchoeneBerg, Elliptic modular functions: an introduction. (Translated from the German) Die Grundlehren der mathematischen Wissenschaften, Band 203. Springer-Verlag, New York-Heidelberg, 1974.

6. T. Kubota, Elementary theory of Eisenstein series. Kodansha Ltd., Tokyo; Halsted Press [John Wiley \& Sons], New York-London-Sydney, 1973.

7. H. B. Dwight, Tables of integrals and other mathematical data. 4th ed. The Macmillan Company, New York, 1961.

(Received 20.02.2005)

Author's address:

Department of Mathematics No. 3

Georgian Technical University

77, M. Kostava St., Tbilisi 0193

Georgia

E-mail: nika3966@yahoo.com 